\title{
Localized states in strong magnetic field: resonant scattering and the Dicke effect
}

\author{
T. V. Shahbazyan* and S. E. Ulloa \\ Department of Physics and Astronomy, Condensed Matter and Surface Science Program, Ohio \\ University, Athens, OH 45701-2979
}

\begin{abstract}
We study the energy spectrum of a system of localized states coupled to a $2 \mathrm{D}$ electron gas in strong magnetic field. If the energy levels of localized states are close to the electron energy in the plane, the system exhibits a kind of collective behavior analogous to the Dicke effect in optics. The latter manifests itself in "trapping" of electronic states by localized states. At the same time, the electronic density of states develops a gap near the resonance. The gap and the trapping of states appear to be complementary and reflect an intimate relation between the resonant scattering and the Dicke effect. We reveal this relation by presenting the exact solution of the problem for the lowest Landau level. In particular, we show that in the absence of disorder the system undergoes a phase transition at some critical concentration of localized states.

Pacs numbers: 71.27.+a, 73.20.Dx, 72.15.Nj
\end{abstract}

Typeset using REVTEX

${ }^{*}$ Current address: Department of Physics and Astronomy, Vanderbilt University, Nashville, TN 37235 


\section{INTRODUCTION}

Electronic states of two-dimensional (2D) systems in a magnetic field in the presence of impurities have been intensively studied during the last two decades 10 the macroscopic degeneracy of the Landau levels (LL) makes impossible a perturbative treatment of even weak disorder and calls for non-perturbative approaches. For high LL, Ando's self-consistent Born approximation 1 was shown to be asymptotically exact for short-range disorder, 14 while in the case of long-range disorder the averaged density of states (DOS) was obtained within the eikonal approximation.14 For low LL and uncorrelated disorder, the problem contains no small parameter and neither of those approximations applies. Nevertheless, for the lowest LL, the exact DOS in a white-noise potential has been obtained by Wegner by mapping the problem onto that of the 0D complex $\phi^{4}$-model 6 This remarkable result was extended to non-Gaussian distributions of random potential by Brézin, Gross, and Itzykson within the functional-integral approach,

In the works mentioned above, the energy levels of the impurities played no role in the scattering. Experimentally, this is well justified since usually the random potential comes from the charged donors with energy levels substantially higher than the Fermi energy in the plane. The Gaussian form of the distribution function implies that random potential is created by a large number of relatively weakly scattering impurities. The LL shape is then described by a "smooth" curve, symmetric with respect to the LL center. In the case of point-like scatterers with constant scattering strength, the DOS is strongly asymmetric,2 3 , 3 . vanishing below (above) the LL center for repulsive (attractive) potential. An asymmetry, caused by deviations from the Gaussian distribution, has been observed in very low-mobility heterostructures. 17

The situation is quite different in the presence of localized states (LS) with energies close to the electron energy in the plane. A large number of such LS's can have a dramatic effect on the properties of the 2D electron gas. Such experimental structures became available with recent advances in fabrication of arrays of ultra-small InAs quantum dots with unusually 
narrow distribution of parameters 1820 With typical sizes of less than $20 \mathrm{~nm}$ and variations of less that $10 \%$, an array of such dots with density $10^{10}-10^{11} \mathrm{~cm}^{-1}$ can be produced at some preset distance from a plane of high mobility electrons. 21 As the Fermi energy in the plane is brought close to the levels of dots, the scattering becomes strongly enhanced. It was, in fact, observed in Ref. 21 that the mobility dropped by two orders of magnitude when the thickness of the tunneling barrier between the dots and the plane was reduced.

In this paper we study the electronic states of a system consisting of $2 \mathrm{D}$ electron gas in strong magnetic field and point-like LS's with energy levels close to the electron energy in the plane. It is important to realize that the combined effect of such LS's differs drastically from that of a collection of isolated LS's. The reason lies in a specific type of coupling between LS's, which originates from electronic transitions between LS's and the electron plane. For an isolated LS, such transitions merely lead to broadening of the LS level. However, in the case of many LS's, the electron in the "course" of a single transition between a particular LS and the the plane, "visits" also the other LS's, propagating in the plane between successive transitions. As a result, the LS's become coupled via the states in the plane. This coupling differs qualitatively from the usual overlap of the LS wave-functions, and leads to formation of certain coherent state.22

Let us illustrate the role of such coupling between LS's (in the absence of magnetic field) on the following example. Consider first an isolated LS with energy $\epsilon_{1}$. In the absence of tunneling, the spectral function (SF) of the LS is simply $S_{0}(\omega)=\delta\left(\omega-\epsilon_{1}\right)$. Turning on the tunneling transforms the SF into the Lorentzian

$$
S(\omega)=-\frac{1}{\pi} \operatorname{Im} \frac{1}{\omega-\epsilon_{1}+i W}=\frac{1}{\pi} \frac{W}{\left(\omega-\epsilon_{1}\right)^{2}+W^{2}},
$$

with

$$
W=\pi \sum_{\mathbf{k}}\left|t_{1 \mathbf{k}}\right|^{2} \delta\left(\omega-E_{\mathbf{k}}\right),
$$

where $t_{1 \mathbf{k}}$ is the amplitude of tunneling between the LS and state $\mathbf{k}$ in the plane. The meaning of Eq. (II) is, of course, that in the presence of tunneling the LS level acquires a width, $W^{-1}=\tau$ being the decay time (we set $\hbar=1$ ). 
Let us now place another LS with energy $\epsilon_{2}$ at some distance from the LS 1. Then a simple generalization of Eq. (1) gives

$$
S(\omega)=-\frac{1}{\pi} \operatorname{Im} \frac{1}{2} \operatorname{Tr} \frac{1}{\omega-\hat{\epsilon}+i \hat{W}},
$$

where $\hat{\epsilon}$ is diagonal $2 \times 2$ matrix with eigenvalues $\epsilon_{1}$ and $\epsilon_{2}$, and

$$
W_{i j}=\pi \sum_{\mathbf{k}} t_{i \mathbf{k}} t_{\mathbf{k} j} \delta\left(\omega-E_{\mathbf{k}}\right)
$$

is the matrix of widths. The key observation is that the matrix elements of $\hat{W}$ are not independent, but instead satisfy a certain relation.22 This relation follows from the definition of $t_{i \mathbf{k}}$ as the overlap between wave functions of the LS $i$ and of the state $\mathbf{k}$ in the plane. Since the latter is simply a plane wave, $t_{i \mathbf{k}}$ contains a phase factor depending on the inplane coordinate $\mathbf{r}_{i}$ of the LS: $t_{i \mathbf{k}}=e^{i \mathbf{k} \cdot \mathbf{r}_{i}} t_{i}$, with $|\mathbf{k}|=k_{F}=2 \pi / \lambda_{F}, \lambda_{F}$ being the Fermi wave length. For diagonal elements, $W_{i i} \equiv W_{i}$, the product $t_{i \mathbf{k}} t_{\mathbf{k} i}$ in Eq. (四) is independent of orientation of $\mathbf{k}$. For non-diagonal elements, however, the product $t_{i \mathbf{k}} t_{\mathbf{k} j}$ contains the factor $e^{i \mathbf{k} \cdot \mathbf{r}_{i j}}, r_{i j}$ being the distance between the LS's. One then obtains from Eq. (4)

$$
W_{12}=q \sqrt{W_{1} W_{2}}, \quad q=J_{0}\left(r_{12} k_{F}\right),
$$

where $J_{0}(x)$ is the Bessel function. For the simplest case of identical LS's, $\epsilon_{i}=\epsilon, W_{i}=W$ and $W_{12}=q W$, Eq. (3) yields

$$
S(\omega)=\frac{1}{\pi}\left[\frac{1}{2} \frac{W_{-}}{(\omega-\epsilon)^{2}+W_{-}^{2}}+\frac{1}{2} \frac{W_{+}}{(\omega-\epsilon)^{2}+W_{+}^{2}}\right],
$$

with $W_{ \pm}=(1 \pm q) W$.

It can be seen that if the two LS's are well separated, $r_{12} k_{F} \gg 1$, then the parameter $q$ is small and the SF again has the form of simple Lorentzian with the width $W$. However, if the distance between LS's is smaller than the Fermi wave length, $r_{12} k_{F} \lesssim 1$, then $q \sim 1$ and both diagonal and non-diagonal elements of $\hat{W}$ are of the same order of magnitude. The SF then represents a superposition of a narrow and a broad Lorentzian with widths $W_{-}$ and $W_{+}$, respectively. This, in turn, gives rise to a short, $\tau_{+}=W_{+}^{-1}=\tau /(1+q)$, and long, 
$\tau_{-}=W_{-}^{-1}=\tau /(1-q)$, decay times. In other words, the state formed by two LS's, coupled via the continuum of states in the plane, is split into fast and slow decaying components.22

The physical mechanism leading to the appearance of fast and slow decaying components is, in fact, analogous to that of the Dicke effect in the spontaneous emission of light by a gas.23 In particular, the case of two LS's, coupled via the continuum of electronic states with $\lambda_{F} / r_{12} \gg 1$, is similar to the case of a pair of atoms radiating a photon with the wave length $\lambda$ much larger than interatomic distance $d$. For $\lambda / d \gg 1$, which corresponds to the limit $q \rightarrow 1$, the two atoms form a single quantum-mechanical system. The electromagnetic field couples only to the symmetric state, which is the fast-radiating component, whereas for the antisymmetric state (slow-radiating component) the corresponding matrix element vanishes. The emission spectrum represents a narrow peak with the width $W_{-} \rightarrow 0$ on top of a wide peak with the width $W_{+} \rightarrow 2 W$, where here $W^{-1}=\tau$ is the radiating time of an isolated atom. The wide peak, corresponding to the fast time $\tau_{+}=\tau / 2$, is a manifestation of the superradiance, that is coherent emission with the doubled rate, while the narrow peak, corresponding to the subradiance, describes the trapping of radiation by atoms.24 Similarly, the first term of the SF (6), which turns into $\frac{1}{2} S_{0}(\omega)=\frac{1}{2} \delta(\omega-\epsilon)$ for $r_{12} k_{F} \ll 1$, describes the trapping of electronic states by the LS's ("subtunneling"), and the second term indicates that the fast component decays into the continuum of states in the plane with the doubled rate ("supertunneling").

This analogy holds for an arbitrary number of LS's. The SF of $N$ LS's is still given by Eqs. (3) (with factor $1 / 2$ replaced by $1 / N$ ) and (4), where $\hat{W}$ and $\hat{\epsilon}$ are now $N \times N$ matrices. For identical LS's confined within the area $\lambda_{F}^{2}$, all the elements of $\hat{W}$ are again of the same order of magnitude. To gain qualitative understanding, let us assume them equal, $W_{i j}=q W$, with some $q \sim 1$. Then the generalization of Eq. (6) reads

$$
S(\omega)=\frac{1}{\pi}\left[\left(1-\frac{1}{N}\right) \frac{W_{-}}{(\omega-\epsilon)^{2}+W_{-}^{2}}+\frac{1}{N} \frac{W_{s}}{(\omega-\epsilon)^{2}+W_{s}^{2}}\right],
$$

with $W_{s}=[1+q(N-1)] W$. We see again that as $q \rightarrow 1$, a fraction $1-1 / N$ of all states becomes trapped by the LS's, while the remaining fraction $1 / N$ is distributed in a 
wide interval $N W$. The latter translates into the fast decay time $\tau_{s}=\tau / N$. This is again completely analogous to the Dicke effect for $N$ atoms confined in a volume with linear size much smaller than $\lambda$.

With this understanding, let us turn back to our system of randomly distributed LS's coupled to a $2 \mathrm{D}$ electron gas in strong magnetic field. In a realistic system, in addition to LS's, a "usual" disorder is present in the electron plane, which we assume to be uncorrelated. At the same time, the energy levels of LS's are not all the same, but, in general, distributed within some interval. This introduces into the problem yet another type of disorder, which is completely absent in the Dicke effect for a gas of identical atoms. As we will see below, the interplay of the two types of disorder appears to be rather non-trivial.

An important parameter characterizing the system is the number of LS's in the area $\lambda_{F}^{2}$. For the lowest LL, this parameter is just the "filling factor" of LS's,

$$
\nu=\left(2 \pi l^{2}\right) n_{L S}
$$

where $n_{L S}$ is LS's concentration and $l$ is the magnetic length. For $\nu \ll 1$, the coupling between LS's via the states in the plane is weak, and $S(\omega)$ represents a convolution of SF's of isolated LS's (coupled to the plane). In the opposite limit, $\nu \gg 1$, the coupling between LS's is strong and, as the above example suggests, nearly all electronic states should be trapped by the LS's. Note however that in this example, the collective (Dicke) state is characterized by the proximity of the parameter $q$ to unity: in the limit $q \rightarrow 1$, the fraction $1-1 / N$ of electronic states is trapped for arbitrary $N$ (which is analogous to $\nu$ ). Although, it is not possible to introduce a priori a parameter similar to $q$ in a disordered system, one expects on physical grounds that with increasing $\nu$ the system will find itself in the Dicke state. In particular, for large $\nu$, one expects that the weight $1-1 / \nu$ of the $\mathrm{SF} S(\omega)$ will be carried by the "bare" SF, $S_{0}(\omega)$, calculated in the absence of tunneling. However, the interval of $\nu$ within which the crossover between two regimes occurs, should depend strongly on the disorder. As we show below, under certain conditions the transition to the Dicke state can occur at some critical value of $\nu$. 
It is useful to view this system from a slightly different angle. Namely, let us consider the effect of LS's on the electronic states in the plane. Clearly, as the electron energy approaches the LS levels, the electron experiences resonant scattering by the LS's. As a result, the electronic DOS should exhibit a sharp energy dependence near the resonance. The character of this dependence can be easily understood from the physical picture outlined above. Since with increasing $\nu$, a larger fraction of the electronic states is trapped by the LS's, the DOS should develop a gap in the limit of large $\nu$. Incidentally, the fact that resonant scattering should lead to a gap for large LS's concentrations has long been known in the 3D case for identical scatterers (in the absence of magnetic field).25 20 The above arguments suggest that the resonant scattering and the Dicke effect are, in a certain sense, complementary to each other. The goal of the present paper is to establish this relation in precise terms.

In fact, it is easy to see that the shape of the SF of LS's is determined entirely by the resonant scattering. Indeed, the electron in the "course" of a single transition between a LS and the plane is being scattered by the rest of LS's. Therefore, the self-energy of a LS is simply proportional to the Green function of $2 \mathrm{D}$ electron in the presence of resonant scattering. This formal relation indicates, however, that finding the SF, averaged over the positions and energies of LS's as well as over the in-plane disorder, is, in general, a rather difficult task. In particular, it requires the calculation of not only the averaged electron Green function 28 but, in fact, of all its moments. Nevertheless, as we show below, for the lowest LL level the problem can be solved exactly. This solution, which is the main result of the present paper, is possible due to the hidden supersymmetry of the lowest LL.

Let us briefly summarize our results. The exact expressions for the SF $S(\omega)$, and the DOS $g(\omega)$, are multiparametric functions determined by the LS filling factor $\nu$, the tunneling strength $\delta$, the Wegner's width $\Gamma$ of the lowest LL (characterizing the in-plane disorder), and the distribution function of the LS levels $f_{\gamma}(\epsilon-\bar{\epsilon})$, where $\bar{\epsilon}$ is the average energy and $\gamma$ is the width. In the absence of coupling to the plane, $\delta=0$, the "bare" SF is simply $S_{0}(\omega)=f_{\gamma}(\omega-\bar{\epsilon})$. In the presence of coupling, $\delta \neq 0$, we distinguish between two regimes 
governed by the dimensionless parameter $\delta^{2} / \gamma \Gamma$.

In the weak coupling regime, $\delta^{2} / \gamma \Gamma \ll 1$, we find that the transition to the Dicke state is smooth. In the limit of large $\nu$, the fraction $1-1 / \nu$ of electronic states is trapped by the LS's, yielding $S(\omega)=(1-1 / \nu) S_{0}(\omega)$. At the same time, the DOS in the presence of resonant scattering exhibits a pronounced minimum which develops into a gap with increasing $\nu$. The width of the gap is independent of the disorder and is determined by the tunneling strength and the LS concentration only. We demonstrate that this behavior is universal and persists for arbitrary distribution of LS levels.

In the strong coupling regime, $\delta^{2} / \gamma \Gamma \gg 1$, the SF and the DOS exhibit a rather complicated behavior. In the limit of vanishing in-plane disorder and LS level spread, both $S(\omega)$ and $g(\omega)$ are non-analytic in $\omega$, turning to zero in a finite energy interval. This gap originates from the infinite degeneracy of the LL in the absence of disorder and is unrelated to the gap in the weak coupling regime due to the trapping of states.

We find that in the case of strong coupling, the transition to the Dicke state occurs at the critical concentration of LS's corresponding to $\nu=1$ : for arbitrary $\nu>1$, a fraction $1-1 / \nu$ of states is trapped by LS's. At the same time, the DOS exhibits a seemingly similar behavior for $\nu<1$ : a fraction $1-\nu$ of states in the LL center remains unaffected by the resonant scattering. The origin of such "condensation of states" is analogous to the one in the case of non-resonant point-like scatterers. 目䀢 For $\nu<1$, one can choose as a basis linear combinations of unperturbed wave functions vanishing at the positions of all LS's. This reduces the LL degeneracy by a factor $1-\nu$, leaving this fraction of states unaffected.

In fact, the similar behavior of $S(\omega)$ for $\nu>1$ and of $g(\omega)$ for $\nu<1$ is not coincidental, but is a consequence of a rather remarkable relation between the SF and the DOS in the absence of disorder. We demonstrate that $S(\omega)$ and $g(\omega)$ turn into each other under the transformation $\nu \leftrightarrow 1 / \nu$ and $\omega \leftrightarrow \epsilon-\omega$. This unexpected "duality" relates to each other the two phase transitions of entirely different physical origins.

The paper is organized as follows. In Section II we formulate the model and derive the general expression for the SF. In Section III the calculation of the averaged Green function 
of LS's is performed. The analysis and numerical results are presented in Section IV. Section $\mathrm{V}$ concludes the paper.

\section{LOCALIZED STATES AND RESONANT SCATTERING}

Consider a 2D electron gas in strong perpendicular magnetic field in the presence of Gaussian random potential $V(\mathbf{r})$ with correlator

$$
\overline{V(\mathbf{r}) V\left(\mathbf{r}^{\prime}\right)}=w \delta\left(\mathbf{r}-\mathbf{r}^{\prime}\right)
$$

The electron plane is separated by a tunneling barrier from a plane of point-like LS's. We assume that the energy levels of LS's are close to the lowest LL and adopt the tunneling Hamiltonian

$$
\hat{H}=\sum_{\mu} \epsilon_{\mu} a_{\mu}^{\dagger} a_{\mu}+\sum_{i} \epsilon_{i} c_{i}^{\dagger} c_{i}+\sum_{\mu, i}\left(t_{\mu i} a_{\mu}^{\dagger} c_{i}+\text { h.c. }\right)
$$

Here $\epsilon_{\mu}, a_{\mu}^{\dagger}$ and $a_{\mu}$ are the eigenenergy, creation and annihilation operators of the eigenstate $|\mu\rangle$ of the Hamiltonian $H_{0}+V(\mathbf{r}), H_{0}$ being the Hamiltonian of free $2 \mathrm{D}$ electron in a magnetic

field, $\epsilon_{i}, c_{i}^{\dagger}$ and $c_{i}$ are those for the $i$ th localized state, and $t_{\mu i}$ is the tunneling matrix element. The latter is defined as

$$
t_{\mu i}=\int d \mathbf{r} d z \psi_{\mu}^{*}(\mathbf{r}, z) V_{i}(\mathbf{r}, z) \psi_{i}(\mathbf{r}, z) \simeq \psi_{\mu}^{*}\left(\mathbf{r}_{i}, z_{i}\right) \int d \mathbf{r} d z V_{i}(\mathbf{r}, z) \psi_{i}(\mathbf{r}, z)
$$

where $V_{i}(\mathbf{r}, z)$ is the LS potential and $\psi_{i}(\mathbf{r}, z)$ is its wave function. In the perpendicular direction, the wave function $\psi_{\mu}^{*}(\mathbf{r}, z)$ decays as $e^{-\kappa z}, \kappa$ being the decay constant, while in the plane it behaves as eigenfunction $\psi_{\mu}^{*}(\mathbf{r})$ of the Hamiltonian $H_{0}+V(\mathbf{r})$. We assume that the tunneling barrier is high enough, so that the dependence of $\kappa$ on $\mu$ can be neglected.22 Thus, we have

$$
t_{\mu i}=\psi_{\mu}^{*}\left(\mathbf{r}_{i}, 0\right) e^{-\kappa z_{i}} \int d \mathbf{r} d z V_{i}(\mathbf{r}, z) \psi_{i}(\mathbf{r}, z)=\psi_{\mu}^{*}\left(\mathbf{r}_{i}\right) t_{i}
$$

with $t_{i}$ determined by the transparency of the barrier.

We are interested in the LS Green function, 


$$
D(\omega)=\overline{N^{-1} \sum_{i}\left\langle i\left|(\omega-\hat{H})^{-1}\right| i\right\rangle}=\overline{N^{-1} \sum_{i} D_{i}(\omega)}
$$

where the overbar stands for averaging over positions and energies of LS's as well as over the random potential $V$. Each term in the sum (13) can be presented as

$$
D_{i}(\omega)=\frac{1}{\omega-\epsilon_{i}-\Sigma_{i}(\omega)}
$$

where $\Sigma_{i}(\omega)$ is the self-energy resulting from a virtual transition between the $i$ th LS and the plane. In the presence of several LS's, such a transition includes also transitions between the plane and the rest of LS's. The latter transitions result in coupling between LS's via the states in the plane. Introducing the coupling matrix $\hat{T}$,

$$
T_{i j}(\omega)=\sum_{\mu} \frac{t_{i \mu} t_{\mu j}}{\omega-\epsilon_{\mu}}
$$

the self-energy $\Sigma_{i}(\omega)$ can be presented as

$$
\Sigma_{i}(\omega)=T_{i i}+\sum_{j}^{\prime} T_{i j} D_{0 j} T_{j i}+\sum_{j k}^{\prime} T_{i j} D_{0 j} T_{j k} D_{0 k} T_{k i}+\cdots,
$$

where $D_{0 j}(\omega)=\left(\omega-\epsilon_{j}\right)^{-1}$, and the prime indicates that the terms $j, k=i$ in the sums are omitted.

It is convenient to recast $\Sigma_{i}(\omega)$ in a different form. Using Eq. (12), the coupling matrix can be written as

$$
T_{i j}(\omega)=t_{i} t_{j} \tilde{G}\left(\mathbf{r}_{i}, \mathbf{r}_{j}\right)
$$

where $\tilde{G}\left(\mathbf{r}, \mathbf{r}^{\prime}\right)=\left\langle\mathbf{r}\left|\left(\omega-H_{0}-V\right)^{-1}\right| \mathbf{r}^{\prime}\right\rangle$ is the Green function of a $2 \mathrm{D}$ electron in the absence of LS's. After substituting Eq. (17) into Eq. (16), the self-energy takes the form

$$
\begin{aligned}
\Sigma_{i}(\omega)= & t_{i}^{2}\left[\tilde{G}\left(\mathbf{r}_{i}, \mathbf{r}_{i}\right)+\int d \mathbf{r} \tilde{G}\left(\mathbf{r}_{i}, \mathbf{r}\right) U(\omega, \mathbf{r}) \tilde{G}\left(\mathbf{r}, \mathbf{r}_{i}\right)\right. \\
& \left.+\int d \mathbf{r} d \mathbf{r}^{\prime} \tilde{G}\left(\mathbf{r}_{i}, \mathbf{r}\right) U(\omega, \mathbf{r}) \tilde{G}\left(\mathbf{r}, \mathbf{r}^{\prime}\right) U\left(\omega, \mathbf{r}^{\prime}\right) \tilde{G}\left(\mathbf{r}^{\prime}, \mathbf{r}_{i}\right)+\cdots\right]
\end{aligned}
$$

with

$$
U(\omega, \mathbf{r})=\sum_{j} \frac{t_{j}^{2}}{\omega-\epsilon_{j}} \delta\left(\mathbf{r}_{j}-\mathbf{r}\right)
$$


The random potential $U(\omega, \mathbf{r})$ describes resonant scattering of electrons by LS's. It has a form similar to that of the point-like scatterers. The crucial difference, however, is that here the scattering strength depends on the proximity of the electron energy to the LS levels. In particular, the potential (19) changes from repulsive to attractive as the electron energy passes through the resonance. Since the LS positions are random with uniform density $n_{L S}$, the distribution function of $U(\omega)$ is Poissonian. Note that due to the spread in the LS energies $\epsilon_{j}$ and tunneling amplitudes $t_{j}$, the scattering strengths in Eq. (19) are also random.

Finally, after summation of the series (18), the self-energy takes the compact form

$$
\Sigma_{i}(\omega)=t_{i}^{2} G\left(\mathbf{r}_{i}, \mathbf{r}_{i}\right),
$$

where

$$
G\left(\mathbf{r}, \mathbf{r}^{\prime}\right)=\left\langle\mathbf{r}\left|\frac{1}{\omega-H_{0}-V-U(\omega)}\right| \mathbf{r}^{\prime}\right\rangle,
$$

is the Green function of $2 \mathrm{D}$ electron in the presence of resonant scattering.

In the following we assume that the magnetic field is strong and the scattering retains the electron in the lowest LL. While this condition is standard for the white-noise potential, it seems to be more restrictive for the resonant scattering. It should be noted, however, that the scattering strength is effectively reduced by the spread in the LS levels. We also assume that the tunneling barrier, separating LS's from the electron plane, is high enough and neglect the difference between tunneling amplitudes of different LS's, setting $t_{i}=t$ in the rest of the paper.

Equations (13), (14), (20) and (21) determine, in principle, the spectral function,

$$
S(\omega)=-\frac{1}{\pi} \operatorname{Im} D(\omega)=-\frac{1}{\pi} \operatorname{Im} \overline{D_{i}(\omega)} .
$$

The averaged LS Green function, $D(\omega)=\overline{D_{i}(\omega)}$, can be presented as the series

$$
\overline{D_{i}(\omega)}=\overline{\frac{1}{\omega-\epsilon_{i}-t^{2} G\left(\mathbf{r}_{i}, \mathbf{r}_{i}\right)}}=\sum_{n=0}^{\infty}\left\langle\frac{t^{2 n}}{(\omega-\epsilon)^{n+1}}\right\rangle_{\epsilon} G_{n}(\omega),
$$

where $G_{n}(\omega)=\overline{G^{n}\left(\mathbf{r}_{i}, \mathbf{r}_{i}\right)}$ and $\langle\cdots\rangle_{\epsilon}$ denotes averaging over $\epsilon$. In obtaining Eq. (23) we used the fact that in Eqs. (14) and (18), the contribution of the $i$ th LS into the potential (19) is excluded. 
The ensemble averaging in Eq. (23) should be performed over both random potentials $V$ and $U(\omega)$. Calculation of $S(\omega)$ requires then an averaging of not only the electron Green function (21), but, in fact, of all the moments $G_{n}(\omega)$. Remarkably, for the lowest LL this averaging can be performed exactly by generalizing the approach of Ref. $\square$

\section{CALCULATION OF THE SPECTRAL FUNCTION}

In order to find the moments $G_{n}(\omega)$, we rely on the hidden supersymmetry of the lowest LL.6.7 We start by noting that $G^{n}(\mathbf{r}, \mathbf{r})$ can be presented as a Gaussian functional integral over bosonic fields,

$$
G^{n}(\mathbf{r}, \mathbf{r})=\frac{(-i)^{n}}{n !} Z^{-1} \int \mathcal{D} \varphi \mathcal{D} \bar{\varphi} e^{i S}[\varphi(\mathbf{r}) \bar{\varphi}(\mathbf{r})]^{n}
$$

with the action (here $\left.\omega_{+}=\omega+i 0\right)$

$$
S[\bar{\varphi}, \varphi]=\int d \mathbf{r} \bar{\varphi}(\mathbf{r})\left[\omega_{+}-H_{0}-V-U(\omega)\right] \varphi(\mathbf{r})
$$

The normalization factor $Z^{-1}$ can be written as a fermionic integral, $Z^{-1}=\int \mathcal{D} \chi \mathcal{D} \bar{\chi} e^{i S}$, with the same action $S[\bar{\chi}, \chi]$. The fields $\varphi(\mathbf{r})$ and $\chi(\mathbf{r})$ are then projected onto the lowest LL subspace according to (we measure all energies from the lowest LL)

$$
\left(\omega-H_{0}\right) \varphi(\mathbf{r})=\omega \varphi(\mathbf{r}), \quad\left(\omega-H_{0}\right) \chi(\mathbf{r})=\omega \chi(\mathbf{r})
$$

Choosing the symmetric gauge, this projection is achieved with the representation

$$
\varphi=\left(2 \pi l^{2}\right)^{-1 / 2} e^{-|z|^{2} / 4 l^{2}} u(z), \quad \chi=\left(2 \pi l^{2}\right)^{-1 / 2} e^{-|z|^{2} / 4 l^{2}} v(z)
$$

where the bosonic field $u(z)$ and the fermionic field $v(z)$ are analytic functions of the complex coordinate $z=x+i y$. In terms of projected fields, Eq. (24) takes the form

$$
G^{n}(\mathbf{r}, \mathbf{r})=\frac{(-i)^{n}}{n !} \frac{e^{-n|z|^{2} / 2 l^{2}}}{\left(2 \pi l^{2}\right)^{n}}\left\langle\left[u(z) \bar{u}\left(z^{*}\right)\right]^{n}\right\rangle,
$$

where angular brackets stand for the functional integration $\int \mathcal{D} u \mathcal{D} \bar{u} \mathcal{D} v \mathcal{D} \bar{v} e^{i S}$ with action 


$$
S[u, v]=\int \frac{d^{2} z}{2 \pi l^{2}} e^{-|z|^{2} / 2 l^{2}}(\bar{u} u+\bar{v} v)\left[\omega_{+}-V-U(\omega)\right]
$$

As a next step, one introduces Grassman (anticommuting) coordinates $\theta$ and $\theta^{*}$, satisfying

$$
\int d \theta=\int d \theta^{*}=0, \quad \int d \theta d \theta^{*} \theta^{*} \theta=\pi^{-1}
$$

(normalized such that $\int d^{2} z d^{2} \theta e^{-|z|^{2}-\theta \theta^{*}}=1$ ), which together with the coordinates $z$ and $z^{*}$ form the "superspace" $\xi=(z, \theta)$. One then defines the analytic "superfields"

$$
\begin{aligned}
\Phi(z, \theta) & =u(z)+\theta v(z) / \sqrt{2} l, \\
\bar{\Phi}\left(z^{*}, \theta^{*}\right) & =\bar{u}\left(z^{*}\right)+\theta^{*} \bar{v}\left(z^{*}\right) / \sqrt{2} l,
\end{aligned}
$$

taking values in the "superspace" $\xi$. Using the identities $\langle u\rangle=\langle v\rangle=0,\langle u \bar{u}\rangle=\langle v \bar{v}\rangle$, and $\left\langle(u \bar{u})^{n}\right\rangle=n\langle u \bar{u}\rangle\left\langle(u \bar{u})^{n-1}\right\rangle$, it is readily seen that the following chain of equalities holds

$$
\left\langle(\Phi \bar{\Phi})^{n}\right\rangle=\left\langle(u \bar{u})^{n}\right\rangle+\frac{n^{2} \theta \theta^{*}}{2 l^{2}}\langle v \bar{v}\rangle\left\langle(u \bar{u})^{n-1}\right\rangle=\left(1+\frac{n \theta \theta^{*}}{2 l^{2}}\right)\left\langle(u \bar{u})^{n}\right\rangle=e^{n \theta \theta^{*} / 2 l^{2}}\left\langle(u \bar{u})^{n}\right\rangle .
$$

Thus, the correlation function (28) can be presented in terms of a functional integral over superfields (31) as

$$
G^{n}(\mathbf{r}, \mathbf{r})=\frac{(-i)^{n}}{n !} \frac{e^{-n \xi \xi^{*} / 2 l^{2}}}{\left(2 \pi l^{2}\right)^{n}} \int \mathcal{D} \Phi \mathcal{D} \bar{\Phi} e^{i S}\left[\Phi(\xi) \bar{\Phi}\left(\xi^{*}\right)\right]^{n}
$$

with $\xi \xi^{*} \equiv|z|^{2}+\theta \theta^{*}$. The action $S[\bar{\Phi}, \Phi]$ in Eq. (33) is obtained from Eq. (29) by substituting

$$
\frac{e^{-|z|^{2} / 2 l^{2}}}{2 \pi l^{2}}(\bar{u} u+\bar{v} v)=\int d^{2} \theta e^{-\xi \xi^{*} / 2 l^{2}} \bar{\Phi}\left(\xi^{*}\right) \Phi(\xi) \equiv Q\left(z, z^{*}\right)
$$

We now perform the ensemble averaging over $V$ and $U(\omega)$. The Gaussian averaging of $\exp \left(-i \int V Q d^{2} z\right)$ gives $\exp \left[-(w / 2) \int Q^{2} d^{2} z\right]$. The averaging over the LS potential $U(\omega)$, carried out with the Poissonian distribution function,29 yields

$$
\overline{\exp \left(-i \int U Q d^{2} z\right)}=\exp \left\{-n_{L S} \int\left[1-\left\langle\exp \left(-\frac{i t^{2} Q}{\omega-\epsilon}\right)\right\rangle_{\epsilon}\right] d^{2} z\right\}
$$

As a result, one obtains the following effective action

$$
\begin{aligned}
i \bar{S}[\Phi, \bar{\Phi}]= & i \omega_{+} \int d^{2} \xi \alpha-\frac{\Gamma^{2}}{2} \int \frac{d^{2} z}{2 \pi l^{2}}\left(2 \pi l^{2} \int d^{2} \theta \alpha\right)^{2} \\
& -\nu \int \frac{d^{2} z}{2 \pi l^{2}}\left\{1-\left\langle\exp \left[-\frac{i \delta^{2}}{\omega-\epsilon}\left(2 \pi l^{2} \int d^{2} \theta \alpha\right)\right]\right\rangle_{\epsilon}\right\}
\end{aligned}
$$


with $d^{2} \xi=d^{2} z d^{2} \theta$ and

$$
\alpha\left(\xi, \xi^{*}\right)=e^{-\xi \xi^{*} / 2 l^{2}} \bar{\Phi}\left(\xi^{*}\right) \Phi(\xi) .
$$

Here $\Gamma=\left(w / 2 \pi l^{2}\right)^{1 / 2}$ is Wegner's width of the lowest LL in the absence of LS's, $\nu=$ $\left(2 \pi l^{2}\right) n_{L S}$ is the "filling factor" of LS's, and the parameter $\delta=t /\left(2 \pi l^{2}\right)^{1 / 2}$ characterizes the tunneling.

The action (36) possesses a supersymmetry, characteristic for the lowest LL6. 6 This symmetry between $z$ and $\theta$ coordinates is evident for the first term of Eq. (36). It can be made explicit for the second and the third terms also by making use of the identityl

$$
n\left(2 \pi l^{2} \int d^{2} \theta e^{-\theta \theta^{*} / 2 l^{2}} \bar{\Phi} \Phi\right)^{n}=2 \pi l^{2} \int d^{2} \theta e^{-n \theta \theta^{*} / 2 l^{2}}(\bar{\Phi} \Phi)^{n} .
$$

This allows one to replace any functional of the form $\int d^{2} z f\left(2 \pi l^{2} \int d^{2} \theta \alpha\right)$ by the functional $2 \pi l^{2} \int d^{2} \xi h(\alpha)$ with $\partial h(x) / \partial x=f(x) / x$. The result is a manifestly supersymmetric action $\bar{S}=\int d^{2} \xi \mathcal{A}(\alpha)$, where

$$
i \mathcal{A}(\alpha)=i \omega_{+} \alpha-\frac{\Gamma^{2} \alpha^{2}}{4}-\nu \int_{0}^{\alpha} \frac{d \beta}{\beta}\left[1-\left\langle\exp \left(-\frac{i \delta^{2} \beta}{\omega-\epsilon}\right)\right\rangle_{\epsilon}\right] .
$$

With all three terms now depending on superfields via $\alpha\left(\xi, \xi^{*}\right)$ only, the perturbation series (with respect to the second and third terms) for the moments $\left\langle\left[\Phi(\xi) \bar{\Phi}\left(\xi^{*}\right)\right]^{n}\right\rangle$ drastically simplifies. One notices that transformations of the form

$$
\Phi(\xi) \rightarrow \Phi(\xi-\eta) e^{\xi \eta^{*} / 2 l^{2}-\eta \eta^{*} / 4 l^{2}}
$$

generate translations of $\alpha$ in the superspace, $\alpha\left(\xi, \xi^{*}\right) \rightarrow \alpha\left(\xi-\eta, \xi^{*}-\eta^{*}\right)$, and hence leave the action $\bar{S}=\int d^{2} \xi \mathcal{A}(\alpha)$ invariant. This leads to

$$
\left\langle\left[\Phi(\xi) \bar{\Phi}\left(\eta^{*}\right)\right]^{n}\right\rangle=C_{n} e^{n \xi \eta^{*} / 2 l^{2}}
$$

and from Eq. (33)

$$
G_{n}(\omega)=\frac{(-i)^{n} C_{n}}{n !\left(2 \pi l^{2}\right)^{n}}
$$


where the coefficients $C_{n}$ are $\xi$-independent. For free electrons one has $C_{n}=i^{n} n ! / \omega_{+}^{n}$. With the action (39), the coefficient $C_{1}$ determines the averaged electron Green function, $G(\omega) \equiv G_{1}(\omega)$, in the presence of resonant scattering.28

For an arbitrary $n$, the moments $\left\langle\left[\Phi(\xi) \bar{\Phi}\left(\xi^{*}\right)\right]^{n}\right\rangle$ can be derived exactly by extending the arguments of Ref. 7 to the case of $n$-point correlators. A diagram with $N$ internal lines contains $N$ free propagators of the form $-i\left\langle\Phi(\xi) \bar{\Phi}\left(\eta^{*}\right)\right\rangle_{0}=e^{\xi \eta^{*} / 2 l^{2}} / \omega_{+}$, while the contribution of each vertex is proportional to $e^{-m \zeta \zeta^{*} / 2 l^{2}}, 2 m$ being the number of lines entering the vertex. After extracting a common factor $e^{n \xi \xi^{*} / 2 l^{2}}$, in accordance with Eq. (41), the contribution of a diagram can be written as $c_{N} K_{N}$, where $K_{N}$ is a ( $N$-fold) Gaussian integral in superspace. The value of the latter is unity due to the exact cancellation between $z$ and $\theta$ integrals. The remaining coefficients $c_{N}$ can be generated within the zero-dimensional field theory with partition function $Z_{0}=\int d^{2} \phi e^{i \mathcal{A}\left(\phi \phi^{*}\right)}$, where $\phi$ is a complex number and the action $\mathcal{A}\left(\phi \phi^{*}\right)$ is the same as in Eq. (39). The coefficients $C_{n}$ are then found as ratios of two ordinary integrals

$$
C_{n}=Z_{0}^{-1} \int d^{2} \phi e^{i \mathcal{A}\left(\phi \phi^{*}\right)}\left(\phi \phi^{*}\right)^{n}
$$

With such $C_{n}$ and with help of Eqs. (42) and (23), we finally arrive at the following exact expression for the Green function of LS's

$$
\begin{aligned}
D(\omega) & =\frac{\pi}{Z_{0}} \int_{0}^{\infty} d \alpha e^{i \mathcal{A}(\alpha)}\left\langle\frac{1}{\omega-\epsilon} \exp \left(-\frac{i \delta^{2} \alpha}{\omega-\epsilon}\right)\right\rangle_{\epsilon}, \\
Z_{0} & =\pi \int_{0}^{\infty} d \alpha e^{i \mathcal{A}(\alpha)}
\end{aligned}
$$

with $\mathcal{A}(\alpha)$ given by Eq. (39).

It is also useful to present this expression in a different form. To do this, we introduce the distribution function of LS energies, $f_{\gamma}(\epsilon-\bar{\epsilon})$, where $\bar{\epsilon}$ is the average energy and $\gamma$ is the width. It can be easily seen from Eq. (39) that

$$
\left\langle\frac{1}{\omega-\epsilon} \exp \left(-\frac{i \delta^{2} \alpha}{\omega-\epsilon}\right)\right\rangle_{\epsilon}=D_{0}(\omega)+\frac{i}{\nu} \frac{\partial \mathcal{A}(\alpha)}{\partial \bar{\epsilon}}
$$

where 


$$
D_{0}(\omega) \equiv\left\langle D_{0 j}(\omega)\right\rangle_{\epsilon}=\int d \epsilon \frac{f_{\gamma}(\epsilon)}{\omega-\bar{\epsilon}-\epsilon}
$$

is the averaged LS Green function in the absence of coupling to the electron plane. Combining Eqs. (44) and (45), we finally obtain

$$
D(\omega)=D_{0}(\omega)+\frac{1}{\nu} \frac{\partial \ln Z_{0}}{\partial \bar{\epsilon}}
$$

The analysis of this expression will be performed in the following section.

\section{DISCUSSION AND NUMERICAL RESULTS}

The final expression for the SF, Eq. (22), appears to be rather involved and its analysis

requires distinguishing between several cases. It is convenient to perform explicitly the averaging over $\epsilon$ in the action (39). The result reads3

$$
i \mathcal{A}(\alpha)=i \omega_{+} \alpha-\frac{\Gamma^{2} \alpha^{2}}{4}-\nu \int_{0}^{\infty} \frac{d x}{x} \tilde{f}_{\gamma}(x) e^{i(\omega-\bar{\epsilon}) x}\left[1-J_{0}(2 \delta \sqrt{x \alpha})\right]
$$

where $\tilde{f}_{\gamma}(x)$ is the Fourier transform of the distribution function $f_{\gamma}(\epsilon)$. With such $\mathcal{A}(\alpha)$, one can obtain from Eq. (47)

$$
D(\omega)=-i \int_{0}^{\infty} d x \tilde{f}_{\gamma}(x) e^{i(\omega-\bar{\epsilon}) x}\left\langle J_{0}(2 \delta \sqrt{x \alpha})\right\rangle_{\alpha},
$$

where $\langle\cdots\rangle_{\alpha}$ stands for the average with the partition function $Z_{0}$ from Eq. (44). The electron Green function, $G(\omega)$, is given by a slightly simpler expression

$$
\left(2 \pi l^{2}\right) G(\omega)=-i\langle\alpha\rangle_{\alpha}
$$

which follows from Eqs. (42) and (43) with $n=1$. The form (48) of the action $\mathcal{A}(\alpha)$ introduces the dimensionless parameter $\delta^{2} / \gamma \Gamma$ which represents the relative strength of the coupling between LS's and electronic states in the plane. 


\section{A. Weak coupling}

Consider first the case of weak coupling, $\delta^{2} / \gamma \Gamma \ll 1$. Substituting

$$
\begin{aligned}
\left\langle J_{0}(2 \delta \sqrt{x \alpha})\right\rangle_{\alpha} & =\exp \ln \left\{1-\left[1-\left\langle J_{0}(2 \delta \sqrt{x \alpha})\right\rangle_{\alpha}\right]\right\} \\
& \simeq \exp \left(-\delta^{2}\langle\alpha\rangle_{\alpha} x\right)
\end{aligned}
$$

into the rhs of Eq. (49), we obtain

$$
D(\omega)=\left\langle\frac{1}{\omega-\epsilon-\Sigma(\omega)}\right\rangle_{\epsilon},
$$

with

$$
\Sigma(\omega)=-i \delta^{2}\langle\alpha\rangle_{\alpha}=\delta^{2}\left(2 \pi l^{2}\right) G(\omega)
$$

Thus, the self-energy in this case is proportional to the averaged electron Green function in the presence of resonant scattering. In particular, the width of the SF is determined by the electronic DOS,

$$
g(\omega)=-\frac{1}{\pi} \operatorname{Im} G(\omega)
$$

Note that Eq. (52) could be also readily obtained from Eq. (14) by substituting the averaged $\overline{\Sigma_{i}(\omega)}=\Sigma(\omega)$ from Eq. (20).

To simplify the analysis, let us assume that the distribution of LS levels is Lorentzian (numerical calculations below are performed with the more realistic Gaussian distribution 31 ). Then the averaging in Eq. (52) can be done analytically, yielding

$$
S(\omega)=\frac{1}{\pi} \frac{\gamma+\gamma_{1}}{\left(\omega-\bar{\epsilon}-\epsilon_{1}\right)^{2}+\left(\gamma+\gamma_{1}\right)^{2}}
$$

with

$$
\begin{aligned}
& \epsilon_{1}(\omega)=\delta^{2}\left(2 \pi l^{2}\right) \operatorname{Re} G(\omega), \\
& \gamma_{1}(\omega)=-\delta^{2}\left(2 \pi l^{2}\right) \operatorname{Im} G(\omega)=\pi \delta^{2}\left(2 \pi l^{2}\right) g(\omega) .
\end{aligned}
$$


Consider first the case of isolated LS, that is $\nu=0$ in Eq. (48). Then we have $G(\omega)=G_{W}(\omega)$, where $G_{W}(\omega)$ is Wegner's Green function.6 This leads to

$$
\begin{aligned}
\epsilon_{1} & =\frac{2 \delta^{2}}{\Gamma}\left[\frac{\omega}{\Gamma}-\frac{2}{\pi} \frac{e^{\omega^{2} / \Gamma^{2}} \int_{0}^{\omega / \Gamma} d t e^{t^{2}}}{1+\left(\frac{2}{\sqrt{\pi}} \int_{0}^{\omega / \Gamma} d t e^{t^{2}}\right)^{2}}\right], \\
\gamma_{1} & =\frac{2 \delta^{2}}{\sqrt{\pi} \Gamma} \frac{e^{\omega^{2} / \Gamma^{2}}}{1+\left(\frac{2}{\sqrt{\pi}} \int_{0}^{\omega / \Gamma} d t e^{t^{2}}\right)^{2}} .
\end{aligned}
$$

The renormalization of the LS energy, $\epsilon_{1}$, is a slow function of $\omega: \epsilon_{1} \simeq 2 \delta^{2} \omega(\pi-2) / \pi \Gamma^{2}$ for $\omega \ll \Gamma$ and $\epsilon_{1} \simeq \delta^{2} / \omega$ for $\omega \gg \Gamma$, so that its role is relatively unimportant. In contrast, the renormalization of the width, $\gamma_{1}$, being proportional to the DOS, is a sharp function of $\omega$ : $\gamma_{1}=2 \delta^{2} / \sqrt{\pi} \Gamma$ for $\omega \ll \Gamma$ and $\gamma_{1}=\left(2 \delta^{2} \sqrt{\pi} \omega^{2} / \Gamma^{3}\right) e^{-\omega^{2} / \Gamma^{2}}$ for $\omega \gg \Gamma$. Therefore, for $\bar{\epsilon} \lesssim \Gamma$, the effective width, $\gamma+\gamma_{1}$, experiences a sharp increase as $\omega$ approaches $\bar{\epsilon}$. This, in turn, leads to a minimum in the SF (see Fig. 1).

Let us now turn to the case of many LS's $(\nu \neq 0)$. The Bessel function in Eq. (48) can be expanded to first order in $\delta^{2} / \gamma \Gamma$, yielding

$$
G(\omega)=G_{W}\left(\omega-\nu \delta^{2} D_{0}\right)
$$

with $D_{0}(\omega)$ given by Eq. (46). Thus, in this case the energy of the electron is shifted by an amount proportional to the average potential $\langle U(\omega, \mathbf{r})\rangle_{\epsilon, \mathbf{r}}=\nu \delta^{2} D_{0}(\omega)$. At the same time, the fluctuations of $U(\omega, \mathbf{r})$, which are described by terms of higher orders in $\delta^{2} / \gamma \Gamma$, are small. In other words, the effect of scattering of the electron by LS's is reduced to that of an effective medium. For the usual, non-resonant scattering ( $\omega$-independent $U$ ), this would merely result in renormalization of the energy by a constant. In the case of resonant scattering, however, the average potential is a complex quantity. Its imaginary part, which originates from the spread in the LS levels, is a sharp function of $\omega$. This affects strongly the shape of the DOS and, in turn, of the SF.

For a low LS concentration, $\nu \delta^{2} / \gamma \Gamma \ll 1$, and for $\omega \sim \bar{\epsilon} \ll \Gamma$, we find that the change in the DOS, $\delta g(\omega)$, is given by 


$$
\frac{\delta g}{g}=\frac{\delta \gamma_{1}}{\gamma_{1}} \simeq-\frac{\pi-2}{\sqrt{\pi}} \frac{\nu \delta^{2} \pi}{\Gamma} S_{0}(\omega)
$$

where $S_{0}(\omega)=\pi^{-1} \operatorname{Im} D_{0}(\omega)$ is the "bare" SF in the absence of coupling to the plane. We see that the DOS in the presence of resonant scattering exhibits a minimum. This, in turn, leads to a maximum in the SF via the renormalization of its width, $\gamma_{1}$. Numerical results for several sets of parameters are shown in Figs. 1 and 2.

With increasing LS concentration, the SF develops a pronounced peak which saturates for large $\nu$ (see Fig. 3). In order to understand this behavior, let us consider the case when the LS concentration is high, $\nu \gg 1$, so that $\nu \delta^{2} / \gamma \Gamma \gg 1$, but at the same time the scattering remains weak. Then, the fluctuations of the random potential $U(\omega)$ are still suppressed, but the argument of $G_{W}$ in Eq. (59) is large. Presenting $G_{W}$ as [see Eq. (50)]

$$
G_{W}\left(\omega-\nu \delta^{2} D_{0}\right)=-\frac{1}{2 \pi l^{2}} \frac{\partial}{\partial \omega_{+}} \ln \int_{0}^{\infty} d \alpha \exp \left[i \alpha\left(\omega_{+}-\nu \delta^{2} D_{0}\right)-\frac{\alpha^{2} \Gamma^{2}}{4}\right],
$$

we notice that for $\omega-\bar{\epsilon} \sim \gamma$ one has $\operatorname{Im} D_{0} \sim 1 / \gamma$, so that the last term in the exponent can be omitted. This gives

$$
G_{W}\left(\omega-\nu \delta^{2} D_{0}\right) \simeq \frac{1}{2 \pi l^{2}} \frac{1}{\omega-\nu \delta^{2} D_{0}(\omega)}
$$

If $\bar{\epsilon}$ is not in the LL tail, the first term in the denominator can be neglected. For the Lorentzian distribution, $D_{0}(\omega)=(\omega-\bar{\epsilon}+i \gamma)^{-1}$, this readily leads to $\epsilon_{1}=(\bar{\epsilon}-\omega) / \nu$ and $\gamma_{1}=\gamma / \nu$, and we obtain

$$
S(\omega)=\frac{1}{\pi\left(1+\nu^{-1}\right)} \frac{\gamma}{(\omega-\bar{\epsilon})^{2}+\gamma^{2}} \simeq\left(1-\frac{1}{\nu}\right) S_{0}(\omega)
$$

We see that for large LS concentration, almost the entire weight of $S(\omega)$ is carried by the "bare" SF, $S_{0}(\omega)$, meaning that the fraction $1-1 / \nu$ of electronic states is trapped by LS's. The remaining $1 / \nu$ fraction of states is carried by the tails, $\omega-\bar{\epsilon} \gg \gamma$, which become longer as $\nu$ increases (see Fig. 3). As was discussed in the Introduction, such form of $S(\omega)$ is a manifestation of the Dicke effect. Clearly, in the case of weak coupling, the transition to the Dicke state is smooth. 
Although Eq. (63) was derived for the Lorentzian form of $f_{\gamma}(\epsilon)$, this behavior persists for an arbitrary distribution of LS levels. Indeed, for $\bar{\epsilon} \lesssim \Gamma$ and $\omega-\bar{\epsilon} \sim \gamma$, Eqs. (52), (53), (59), and (62) yield

$$
D(\omega)=D_{0}\left[\omega+1 / \nu D_{0}(\omega)\right] .
$$

Since for such $\omega$ one has $D_{0}(\omega) \sim 1 / \gamma$, we see that $S(\omega)$ is again given by $S_{0}(\omega)$ up to a small fraction $1 / \nu$.

At the same time, with increasing $\nu$ the DOS exhibits a pronounced minimum which turns into a gap as $\nu$ becomes large (see Fig. 4). For $\nu \gg 1$, the width of the gap is of the order of the separation between peaks. The latter can be estimated from the condition that the argument of the Green function (61) turns to zero. Since $D_{0}(\omega) \simeq 1 / \omega$ for large $\omega$, one easily finds that this separation is $2 \sqrt{\nu} \delta$. Thus, the width of the gap is universal and independent of the disorder. Within the gap, the DOS can be estimated from Eq. (62) as $\left(2 \pi l^{2}\right) g(\omega) \sim \gamma / \nu \delta^{2}$.

\section{B. Strong coupling}

In the case of strong coupling, $\delta^{2} / \gamma \Gamma \gg 1$, Eqs. (52) and (53) do not apply and, in general, all moments $G_{n}$ contribute to $D(\omega)$. Nevertheless, the SF and the DOS appear to be intimately related. In order to reveal this relation, let us consider the limit of vanishing disorder, with $\Gamma / \delta \ll 1$ and $\gamma / \delta \ll 1$. In this case the second term in the action (39) can be omitted and no energy averaging is implied. Then it is easy to see that in the energy interval $\omega(\epsilon-\omega)>0$, the integration path in the $\alpha$-integral for the partition function, $Z_{0}=\pi \int_{0}^{\infty} d \alpha e^{i \mathcal{A}(\alpha)}$, can be rotated by $e^{-i \pi \operatorname{sgn}(\omega-\epsilon) / 2}$, resulting in purely real $i \mathcal{A}$. After rescaling the integration variable $\alpha$, the partition function takes the form

$$
Z_{0}=\frac{\pi(\omega-\epsilon)}{i \delta^{2}} \int_{0}^{\infty} d \alpha \exp \left[-\alpha \frac{\omega(\epsilon-\omega)}{\delta^{2}}-\nu \int_{0}^{\alpha} \frac{d \beta}{\beta}\left(1-e^{-\beta}\right)\right] .
$$

With this $Z_{0}$, one obtains from Eq. (47) after some algebra32 


$$
D(\omega)=\left(1-\frac{1}{\nu}\right) \frac{1}{\omega-\epsilon}+\frac{1}{\nu} \frac{\partial}{\partial \epsilon} \ln \int_{0}^{\infty} \frac{d \alpha}{\alpha^{\nu}} \exp \left[-\alpha \frac{\omega(\epsilon-\omega)}{\delta^{2}}-\nu \int_{\alpha}^{\infty} \frac{d \beta}{\beta} e^{-\beta}\right] .
$$

The second term can be written as $-\left(\omega / \delta^{2} \nu\right)\langle\alpha\rangle_{\alpha}$ [calculated with the partition function (65)]. On the other hand, the same manipulations with the electron Green function (50) give $G(\omega)=\left[(\epsilon-\omega) / \delta^{2} 2 \pi l^{2}\right]\langle\alpha\rangle_{\alpha}$. This leads to the following relation

$$
\nu(\epsilon-\omega) D(\omega)+\omega\left(2 \pi l^{2}\right) G(\omega)=1-\nu .
$$

The fact that $\operatorname{Im} \ln Z_{0}$ has no energy dependence for $\omega(\epsilon-\omega)>0$ implies that both the $\mathrm{SF}$ and the DOS should exhibit a gap in this energy interval (see Fig. 5). However, in contrast to the weak coupling case, here the gap is not related to the Dicke effect. The physical origin of this gap can be understood from the following reasoning. ${ }^{28}$ In the absence of in-plane disorder (small $\Gamma$ ), the LL broadening comes from the resonant scattering alone. Then the scattering potential (19) appears to be attractive for $\omega<\epsilon$, pulling the states from the LL center to the left, while for $\omega>\epsilon$ it is repulsive, pushing the states to the right. At the same time, in the absence of LS level spread (small $\gamma$ ), the finite width of $S(\omega)$ comes from transitions between the LS's and the electron plane. Therefore, the absence of states in the latter leaves $S(\omega)$ unaffected [that is $S(\omega)=0$ ] in the same energy interval. It should be emphasized that $S(\omega)$ and $g(\omega)$ are non-analytic, turning to zero for arbitrary $\nu$ (in the weak coupling case, $S(\omega)$ and $g(\omega)$ are finite for all $\omega$, vanishing only in the limit $\nu \rightarrow \infty)$.

Near the gap edges, $\omega(\omega-\epsilon) \rightarrow 0_{+}$, the behavior of $S(\omega)$ and $g(\omega)$ depends strongly on the value of $\nu$. The integral in Eq. (66) is similar to that already analyzed in Ref. 0 . Consider first the case $\nu<1$. The second term in Eq. (66) can be split as

$$
\begin{aligned}
& \frac{1}{\nu} \frac{\partial}{\partial \epsilon}\{(\nu-1) \ln [\omega(\epsilon-\omega)] \\
& \left.+\ln \left[1-\frac{1}{\Gamma(1-\nu)}\left[\frac{\omega(\epsilon-\omega)}{\delta^{2}}\right]^{1-\nu} \int_{0}^{\infty} \frac{d \alpha}{\alpha^{\nu}} e^{-\alpha \omega(\epsilon-\omega) / \delta^{2}}\left[1-\exp \left(-\nu \int_{\alpha}^{\infty} \frac{d \beta}{\beta} e^{-\beta}\right)\right]\right]\right\}
\end{aligned}
$$

where $\Gamma(x)$ is the Gamma-function. The derivative of the first term in the braces cancels the first term in Eq. (66). The second term, after analytical continuation $\omega(\epsilon-\omega) \rightarrow e^{-i \pi} \omega(\omega-\epsilon)$, gives 


$$
S(\omega)=\frac{A}{\nu} \frac{1}{|\omega-\epsilon|^{\nu}}\left|\frac{\omega}{\delta^{2}}\right|^{1-\nu},
$$

where $A$ is a $\nu$-dependent constant $33\left(A \sim \nu^{2}\right.$ as $\left.\nu \rightarrow 0\right)$.

The SF diverges at one edge of the gap, $\omega \rightarrow \epsilon$, and is continuous at the other, $\omega \rightarrow 0$. The exactly opposite behavior is exhibited by the DOS, for which we obtain from Eq. (67)

$$
\left(2 \pi l^{2}\right) g(\omega)=(1-\nu) \delta(\omega)+\nu \frac{\omega-\epsilon}{\omega} S(\omega)
$$

Aside from the first term, the behavior of the DOS near the gap edges, $g(\omega) \propto|\omega|^{-\nu}|\omega-\epsilon|^{1-\nu}$, "mirrors" that of the SF. Figure 5 shows that the similarity is striking over the entire energy range.

The first term in Eq. (70) indicates that a fraction $1-\nu$ of states remains unaffected by the resonant scattering. Such a "condensation of states" originates from the residual LL degeneracy left after arranging the unperturbed wave-functions to vanish at the positions of all LS's, and is similar to that in the case of point-like scatterers with constant scattering strength 2. 3.9 In fact, the analogy extends also to the intricate structure of the DOS away from the gap. In particular, the smaller peaks correspond to the singularities in $g(\omega)$ at integer values of $\omega(\omega-\epsilon) / \delta^{2}$; with increasing $\gamma$ they are washed out. Similar structure appears also in $S(\omega)$; here it is washed out with increasing $\Gamma$ (see Fig. 5).

In the case of $\nu \geq 1$, the DOS at $\omega(\omega-\epsilon) \rightarrow 0_{+}$can be found in a similar manner. The result read\$3

$$
\begin{aligned}
\left(2 \pi l^{2}\right) g(\omega) & \propto \frac{1}{|\omega|} \ln ^{-2}\left[\frac{\omega(\omega-\epsilon)}{\delta^{2}}\right], \quad \text { for } \nu=1 \\
& \propto|\omega|^{\nu-2}\left|\frac{\omega-\epsilon}{\delta^{2}}\right|^{\nu-1}, \quad \text { for } \quad \nu>1 .
\end{aligned}
$$

Note that for $\nu \geq 1$, the LL degeneracy is lifted completely, so that no condensation of states occurs. Instead, according to Eq. (67), the SF represents a sum of two terms

$$
S(\omega)=\left(1-\frac{1}{\nu}\right) \delta(\omega-\epsilon)+\frac{1}{\nu} \frac{\omega}{\omega-\epsilon}\left(2 \pi l^{2}\right) g(\omega)
$$

Since in the absence of disorder $S_{0}(\omega)=\delta(\omega-\epsilon)$, we observe again that the fraction $1-1 / \nu$ of all states is trapped by LS's, while the tails of $S(\omega)$, given by the second term, are 
suppressed by the factor $1 / \nu$. However, in contrast to the weak coupling case, here such behavior persists not only for large, but for arbitrary filling factor $\nu>1$. It is instructive to compare SF from Eq. (72) to the SF from Eq. (7). In the latter case, $N$ identical LS's, confined to the area $\lambda_{F}^{2}$, form a coherent (Dicke) state in the limit $q \rightarrow 1$. In former case, the transition into the Dicke state occurs at critical filling factor $\nu=1$.

Thus, in the absence of disorder, the system undergoes two types of transitions at $\nu=1$ : the condensation of states in the LL center for $\nu<1$, and the trapping of states by LS's for $\nu>1$. It should be emphasized that the two phase transitions have entirely different physical origins and exist independently of each other. The former is caused by the LL degeneracy and persists also for non-resonant scattering; the latter, being a manifestation of the Dicke effect, takes place also in the absence of magnetic field. Nevertheless, the one can be derived from the other due to a surprising "duality" relation. Namely, it is readily seen from Eqs. (170) and (172) that the SF and the DOS turn into each other, $\left(2 \pi l^{2}\right) g(\omega) \leftrightarrow S(\omega)$, under the transformation

$$
\nu \leftrightarrow 1 / \nu, \quad \omega \leftrightarrow \epsilon-\omega
$$

It is rather remarkable that the resonant scattering and the Dicke effect can be unified in such simple manner.

\section{CONCLUSION}

We have shown that a system of LS's coupled to the 2D electron gas in strong magnetic field exhibits a kind of collective behavior similar to the Dicke effect. For high enough LS concentrations, the trapping of electronic states by the LS's takes place, which is analogous to the Dicke subradiance. Such trapping appears to be complementary to the gap in the DOS in the presence of resonant scattering.

Although our derivation was restricted to the lowest LL, we believe that our results are more general and remain valid for higher LL's. There is little doubt that the gap in the 
DOS is a rather general feature. A much more subtle question is related to the type of the transition to the Dicke state. It seems obvious that in a disordered system this transition should be smooth. In a clean system, we have shown that this is, in fact, a phase transition. However, this result appears to be specific to the system in magnetic field, as indicated by the existence of the duality between the trapping of states by LS's and the condensation of states in the LL center. The latter transition, being caused by the LL degeneracy, takes place for all LL numbers 9 (as far as LL mixing is neglected). Therefore, we believe that for higher LL's, the transition to the Dicke state should also occur at critical filling factor $\nu=1$, although we have not proved the duality relation in the general case. In the absence of magnetic field, however, the question remains open.

As a possible experimental realization, we suggest a system of self-assembled quantum dots separated from a 2D electron gas by a tunable tunneling barrier. Due to the ultranarrow distribution of dots' sizes, the spread in their energy levels, $\gamma$, does not exceed 10.0 meV. Although, it is hard to achieve the inter-dot separation much smaller than the Fermi wave-length, a condition $\nu \sim 1$ seems to be quite resonable. For a considerable resonant scattering effect, one has to have $\delta^{2} / \gamma \Gamma \sim 1$. For a typical LL width $\Gamma \sim 1 \mathrm{meV}$, this condition implies that the tunneling parameter $\delta$ should be about several meV. We believe that the significant drop in the mobility, observed (at zero field) by authors of Ref. 21, should be attributed to the gap in the DOS.

\section{ACKNOWLEDGMENTS}

Illuminating discussions with M. E. Raikh are gratefully acknowledged. This work was supported in part by US Department of Energy Grant No. DE-FG02-91ER45334. 


\section{REFERENCES}

${ }^{1}$ T. Ando and Y. Uemura, J. Phys. Soc. Jpn. 36, 959 (1974); T. Ando, A. B. Fowler and F. Stern, Rev. Mod. Phys. 54, 437 (1982).

2 T. Ando, J. Phys. Soc. Jpn. 36, 1521 (1974); 37, 622 (1974); 37, 1233 (1974).

${ }^{3}$ É. M. Baskin, L. N. Magarill and M. V. Éntin, Zh. Eksp. Teor. Fiz. 75, 723 (1978) [Sov. Phys. JETP 48, 365 (1978)].

${ }^{4}$ L. B. Ioffe and A. I. Larkin, Zh. Eksp. Teor. Fiz. 81, 1048 (1981) [Sov. Phys. JETP 54, 556 (1981)].

${ }^{5}$ I. Affleck, J. Phys. C 16, 5839 (1983); 17, 2323 (1984).

${ }^{6}$ F. Wegner, Z. Phys. B 51, 279 (1983).

${ }^{7}$ E. Brézin, D. Gross, and C. Itzykson, Nucl. Phys. B235, 24 (1984).

${ }^{8}$ K. A. Benedict and J. T. Chalker, J. Phys. C 18, 3981 (1985).

${ }^{9}$ K. A. Benedict and J. T. Chalker, J. Phys. C 19, 3587 (1986).

${ }^{10}$ K. A. Benedict, Nucl. Phys. B280, 549 (1987).

11 S. A. Gredeskul, Y. Avishai, and M. Ya Azbel', Europhys. Lett. 21, 489 (1993).

${ }^{12}$ Y. Avishai, M. Ya. Azbel', and S. A. Gredeskul, Phys. Rev. B 48, 17280 (1993).

13 Zusman, Y. Avishai, and S. A. Gredeskul, Phys. Rev. B 48, 17922 (1993).

${ }^{14}$ M. E. Raikh and T. V. Shahbazyan, Phys. Rev. B 47, 1522 (1993).

${ }^{15}$ M. Janßen, O. Viehweger, U. Fastenrath, and J. Hajdu, Introduction to the Theory of the Integer Quantum Hall Effect, (VCH, Weinmheim, 1994).

16 T. V. Shahbazyan and M. E. Raikh, Phys. Rev. Lett. 77, 5106 (1996).

${ }^{17}$ R. J. Haug, R. R. Gerhardts, K. v. Klitzing, and K. Ploog, Phys. Rev. Lett. 59, 1349 
(1987).

${ }^{18}$ D. Leonard, M. Krishnamurthy, C. M. Reaves, S. P. Denbaars, and P. M. Petroff, Appl. Phys. Lett. 63, 3203 (1993); H. Drexler, D. Leonard, W. Hansen, J. P. Kotthaus, and P. M. Petroff, Phys. Rev. Lett. 73, 2252 (1994).

${ }^{19}$ J.-Y. Marzin, J.-M. Gerard, A. Izrael, D. Barrier, G. Bastard, Phys. Rev. Lett. 73, 716 (1994).

${ }^{20}$ M. Grundmann, J. Christen, N. N. Ledentsov, J. Bohrer, D. Bimberg, S. S. Ruvimov, P. Werner, U. Richter, U. Gosele, J. Heydenreich, V. M. Ustinov, A. Yu. Egorov, A. E. Zhukov, P. S. Kop'ev, and Zh. I. Alferov, Phys. Rev. Lett. 74, 4043 (1995).

${ }^{21}$ H. Sakaki, G. Yusa, T. Someya, Y. Ohno, T. Noda, H. Akiyama, Y. Kadoya, and H. Noge Appl. Phys. Lett. 67, 3444 (1995).

${ }^{22}$ T. V. Shahbazyan and M. E. Raikh, Phys. Rev. B 49, 17123 (1994).

${ }^{23}$ R. H. Dicke, Phys. Rev. 93, 99 (1953).

${ }^{24}$ R. G. DeVoe and R. G. Brewer, Phys. Rev. Lett. 76, 2049 (1996).

${ }^{25}$ M. A. Ivanov and Yu. G. Pogorelov Zh. Eksp. Teor. Fiz. 76, 1010 (1979) [Sov. Phys. JETP 49, $510(1979)]$.

${ }^{26}$ I. M. Lifshitz, S. A. Gredeskul, and L. A. Pastur, Introduction to the Theory of Disordered Systems, (Wiley, New York, 1988).

${ }^{27}$ A. P. Jauho and J. W. Wilkins Phys. Rev. B 28, 4628 (1983).

${ }^{28}$ T. V. Shahbazyan and S. E. Ulloa, Phys. Rev. Lett., in press.

${ }^{29}$ R. Friedberg and J. M. Luttinger, Phys. Rev. B 12, 4460 (1975).

${ }^{30}$ Eq. (48) can be obtained with the help of the identity

$$
1-e^{-i \beta \delta^{2} /(\omega-\epsilon)}=\int_{0}^{\infty} d t e^{i(\omega-\epsilon) t^{2} / 4 \beta \delta^{2}} J_{1}(t),
$$


where $J_{1}(t)$ is the Bessel function.

${ }^{31}$ G. Medeiros-Ribeiro, F. G. Pikus, P. M. Petroff, and A. L. Efros, Phys. Rev. B 55, 1568 (1997).

${ }^{32}$ Here one uses the identity

$$
\int_{0}^{\alpha} \frac{d \beta}{\beta}\left(1-e^{-\beta}\right)=C+\ln \alpha+\int_{\alpha}^{\infty} \frac{d \beta}{\beta} e^{-\beta},
$$

where $C$ is the Euler constant.

${ }^{33}$ The $\nu$-dependent constants in Eqs. (69) and (71) are the same as in the Appendix of Ref. 7 


\section{FIGURES}

FIG. 1. The SF at (a) $\nu=0$ (isolated LS), and (b) $\nu=1.5$, with $\Gamma / \gamma=1.0$ and $\bar{\epsilon}=0$, is shown for $\delta / \gamma=0.1$ (dot-dashed line), $\delta / \gamma=0.5$ (long-dashed line), $\delta / \gamma=1.0$ (dashed line), $\delta / \gamma=1.5$ (dotted line), and $\delta / \gamma=2.5$ (solid line).

FIG. 2. The DOS [in units of $g_{1}=\left(2 \pi l^{2}\right)^{-1} \Gamma^{-1}$ ] for a strong in-plane disorder, $\delta / \Gamma=0.3$, with $\bar{\epsilon}=0$ and $\nu=1.5$, is shown for $\gamma / \delta=0.1$ (solid line), $\gamma / \delta=0.5$ (dotted line), $\gamma / \delta=1.0$ (dashed line), $\gamma / \delta=2.0$ (long-dashed line), and $\gamma / \delta=10.0$ (dot-dashed line).

FIG. 3. (a) The SF with $\Gamma / \gamma=1.0, \delta / \gamma=1.0$, and $\bar{\epsilon}=0$ is shown for $\nu=0$ (dot-dashed line), $\nu=0.8$ (long-dashed line), $\nu=3.2$ (dashed line), $\nu=10.0$ (dotted line), and $\nu=16.0$ (solid line). (b) The SF with $\Gamma / \gamma=2.0, \delta / \gamma=2.0$, and $\bar{\epsilon}=0$ is shown for $\nu=0$ (dot-dashed line), $\nu=0.4$ (long-dashed line), $\nu=1.6$ (dashed line), $\nu=6.0$ (dotted line), and $\nu=16.0$ (solid line).

FIG. 4. (a) The DOS with $\gamma / \Gamma=1.0, \delta / \Gamma=1.0$, and $\bar{\epsilon}=0$ is shown for $\nu=0$ (dot-dashed line), $\nu=0.8$ (long-dashed line), $\nu=3.2$ (dashed line), $\nu=10.0$ (dotted line), and $\nu=16.0$ (solid line). (b) The DOS with $\gamma / \Gamma=0.5, \delta / \Gamma=1.0$, and $\bar{\epsilon}=0$ is shown for $\nu=0$ (dot-dashed line), $\nu=0.4$ (long-dashed line), $\nu=1.6$ (dashed line), $\nu=6.0$ (dotted line), and $\nu=16.0$ (solid line).

FIG. 5. (a) The SF for a small LS level spread, $\gamma / \delta=0.1$, with $\nu=0.8$ and $\bar{\epsilon} / \delta=1.0$ is shown for $\Gamma / \delta=0.1$ (solid line), $\Gamma / \delta=0.3$ (dotted line), $\Gamma / \delta=0.5$ (dashed line), and $\Gamma / \delta=1.0$

(long-dashed line). (b) The DOS [in units of $g_{2}=\left(2 \pi l^{2}\right)^{-1} \delta^{-1}$ ] for a weak in-plane disorder, $\Gamma / \delta=0.1$, with $\bar{\epsilon} / \delta=1.0$ and $\nu=0.8$, is shown for $\gamma / \delta=0.1$, (solid line), $\gamma / \delta=0.3$ (dotted line), $\gamma / \delta=0.5$ (dashed line), and $\gamma / \delta=1.0$ (long-dashed line). 


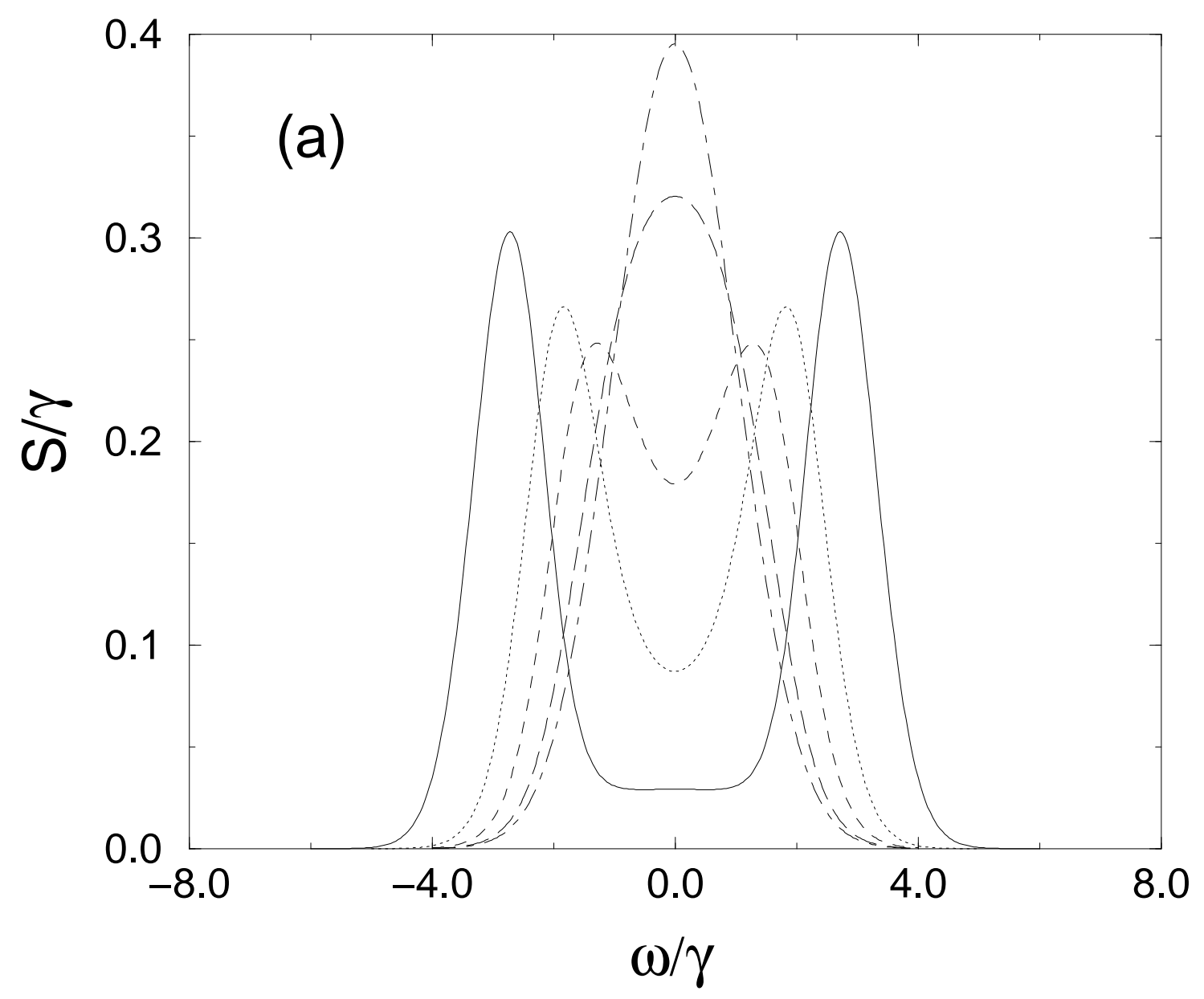

FIG. 1 


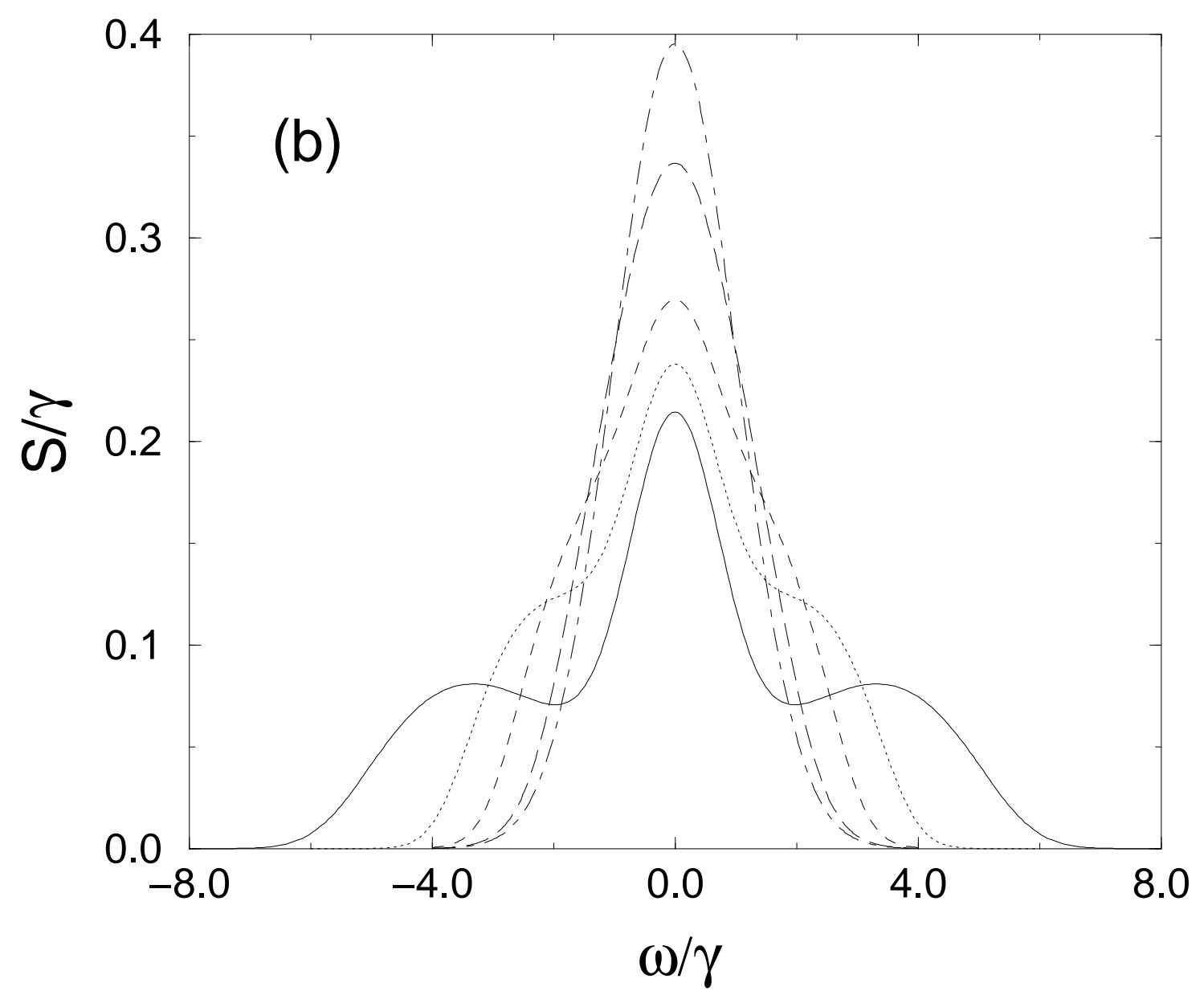

FIG. 1 


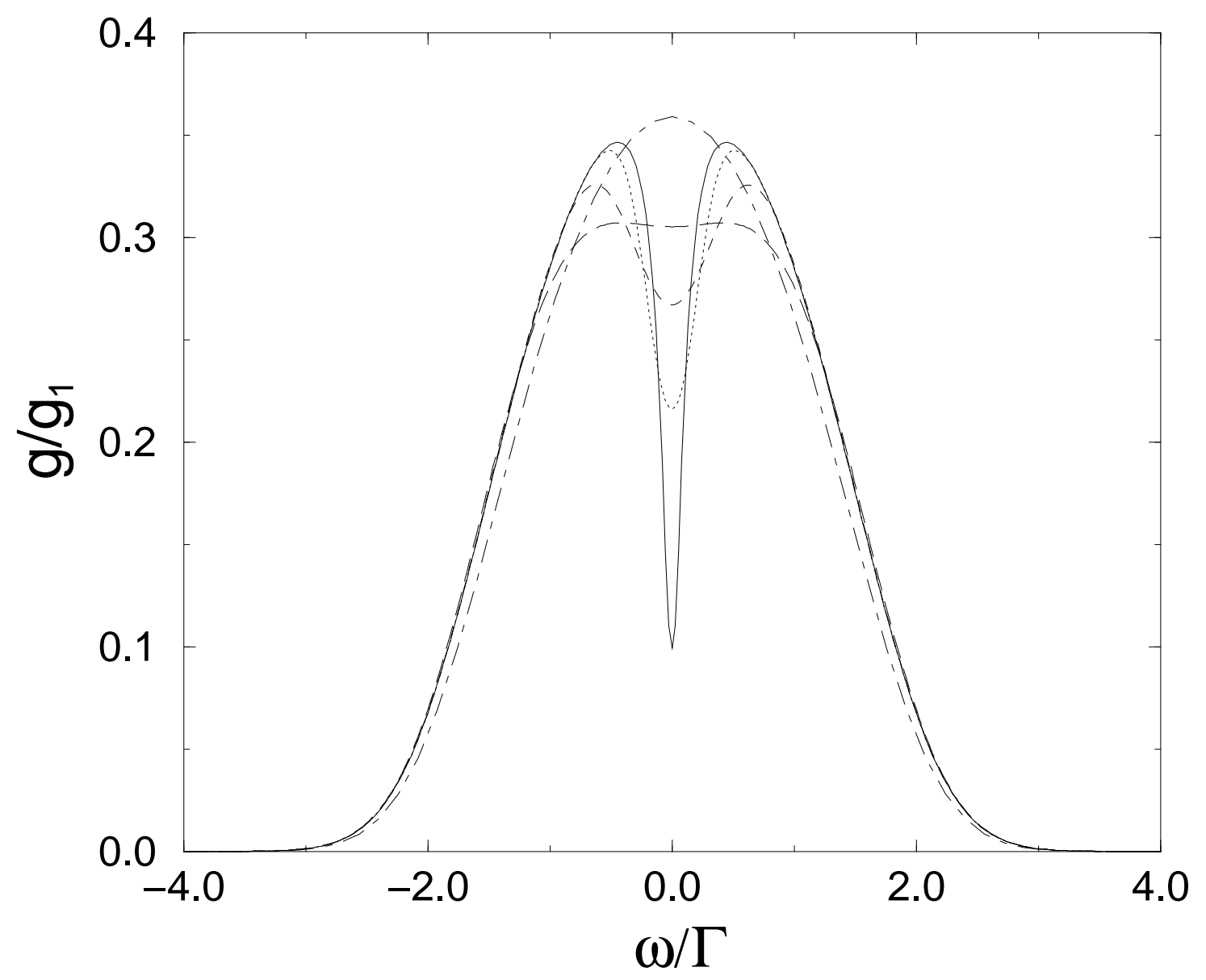

FIG. 2 


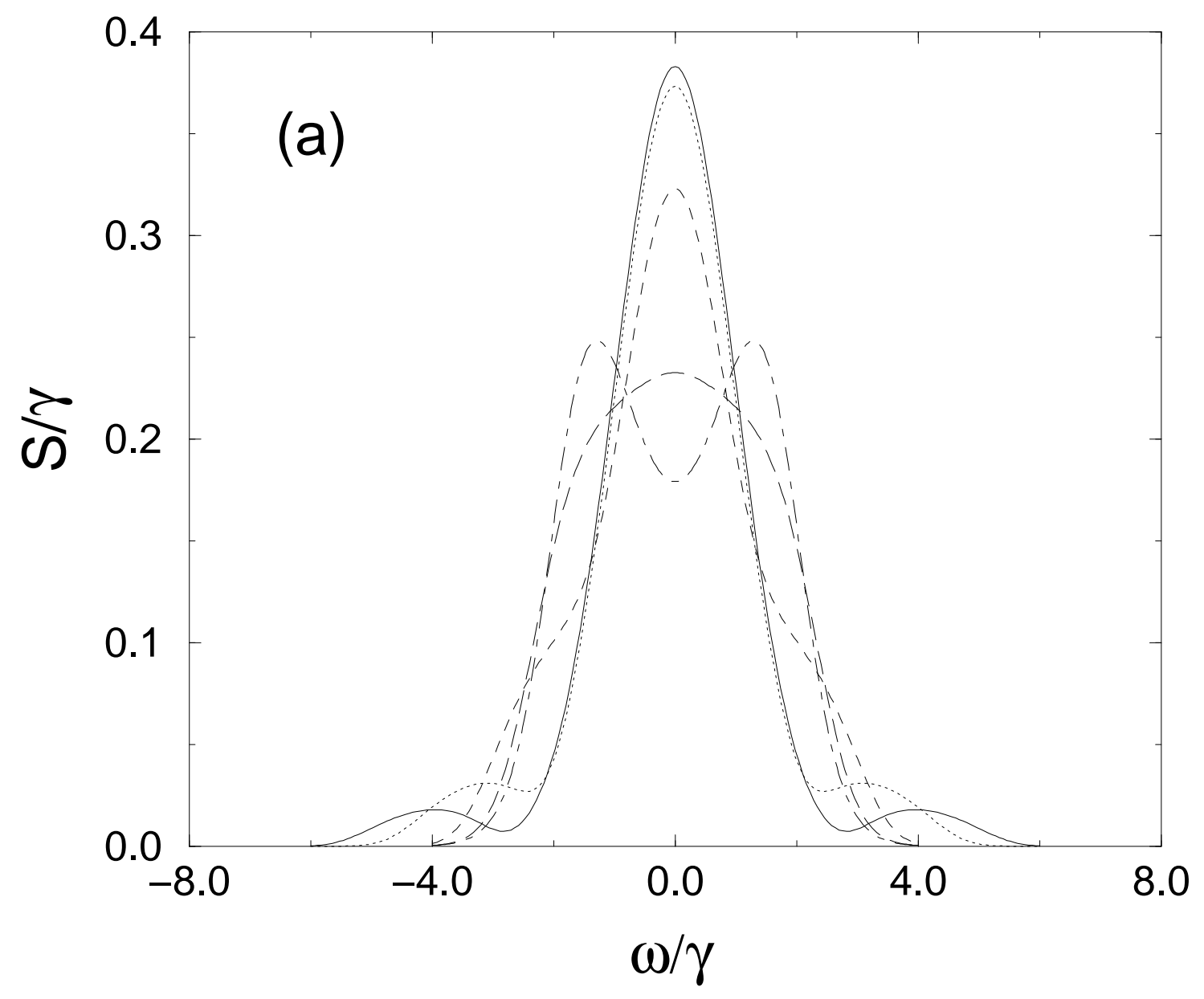

FIG. 3 


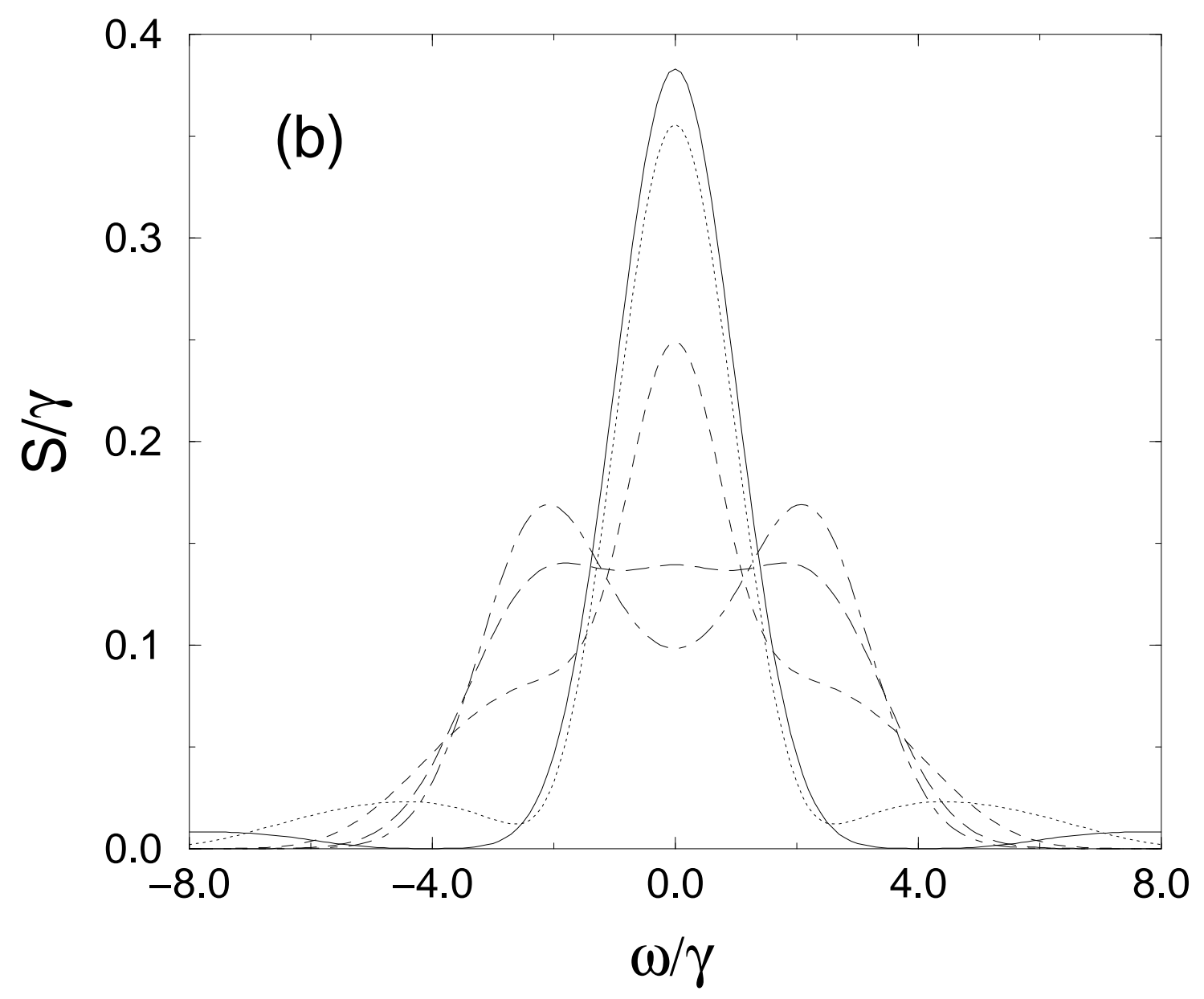

FIG. 3 


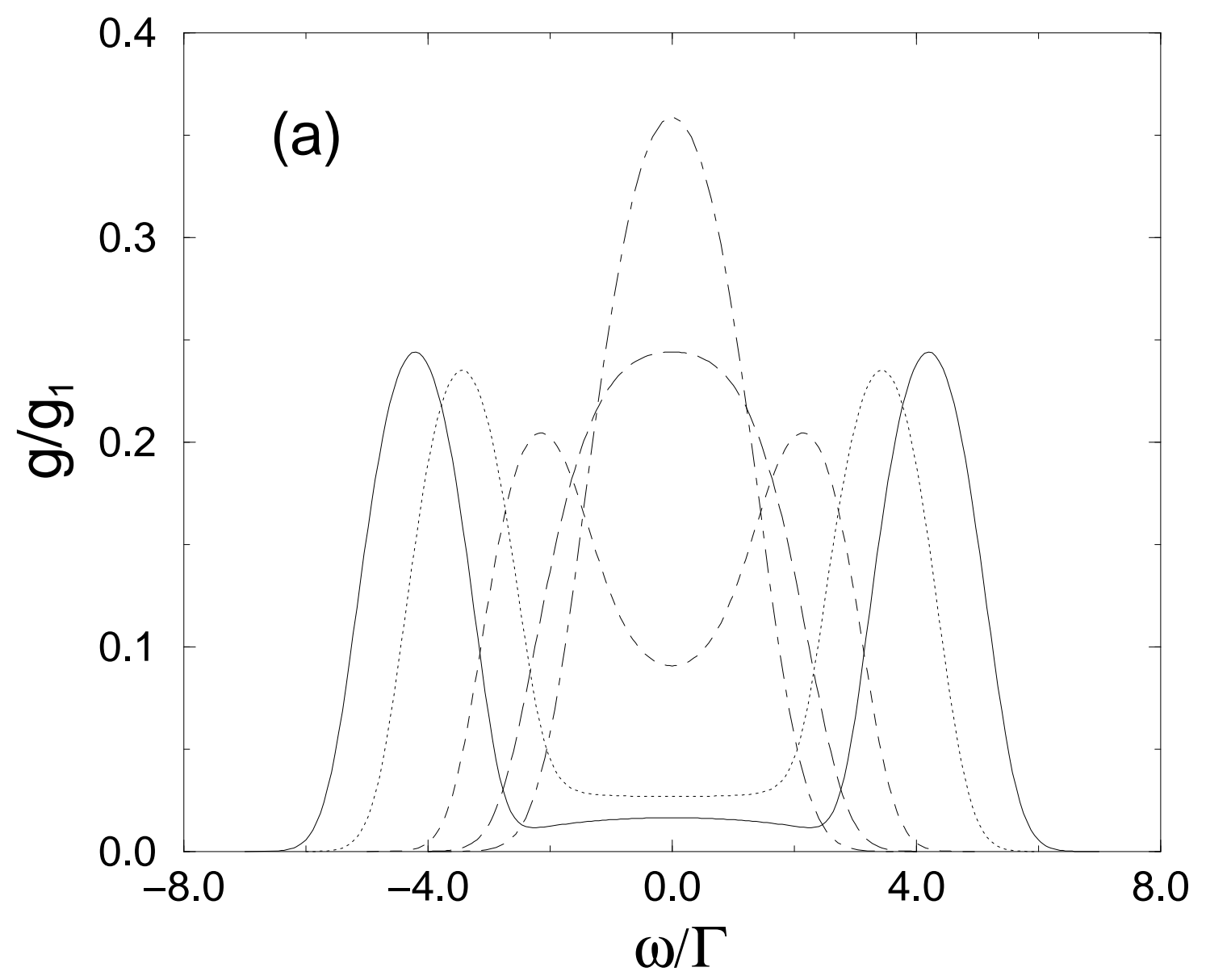

FIG. 4 


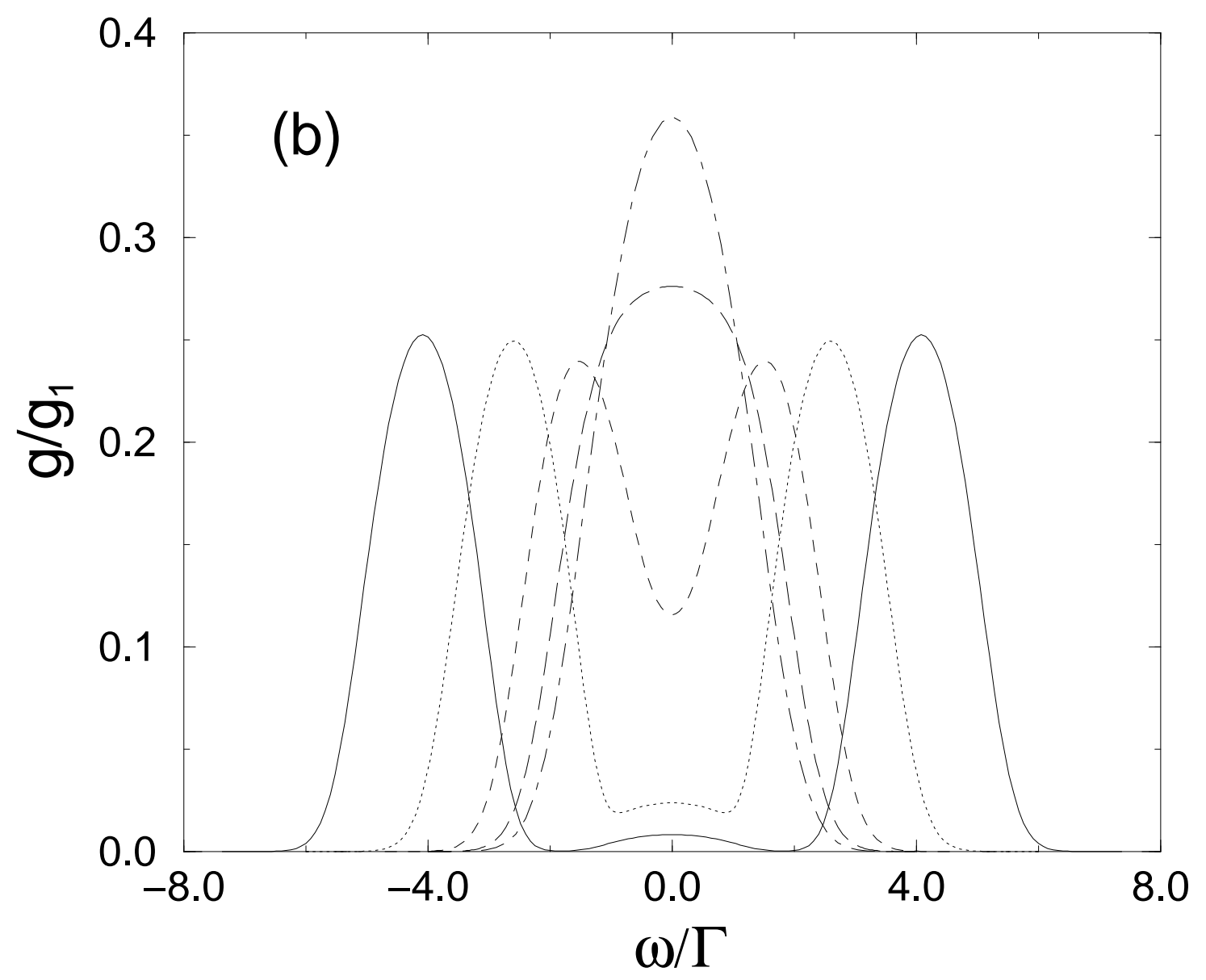

FIG. 4 


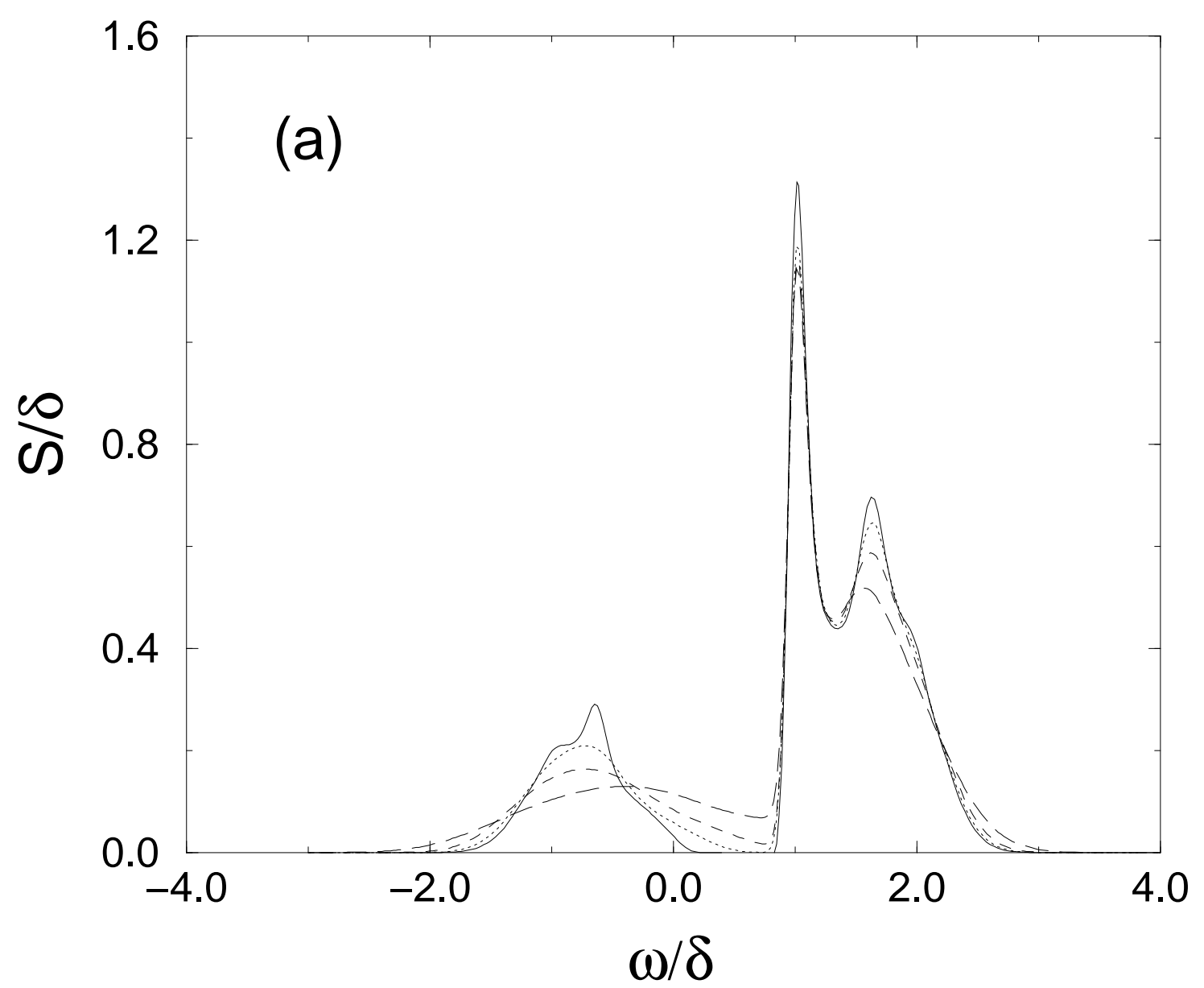

FIG. 5 


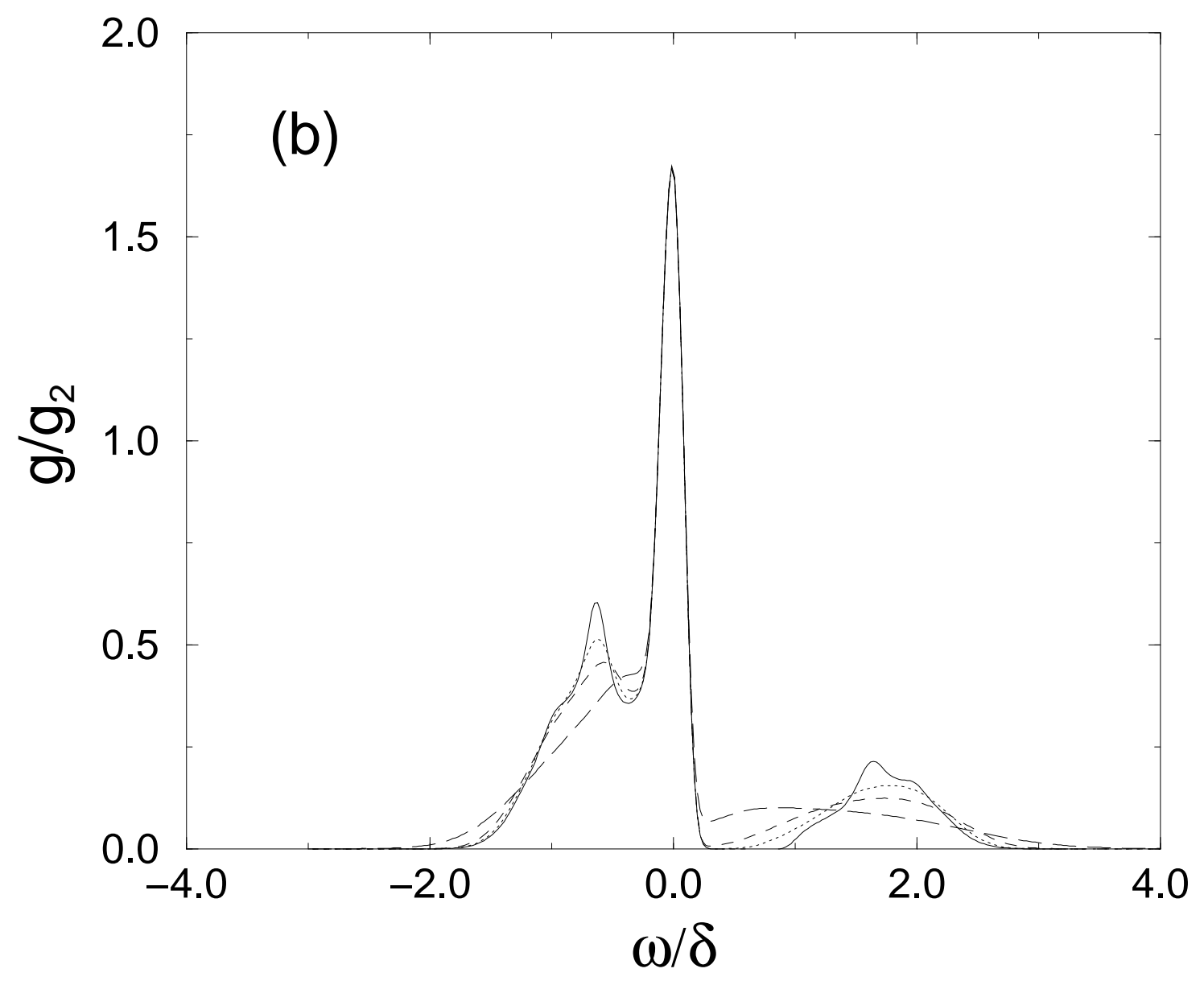

FIG. 5 